\title{
BOUNDARY VALUE PROBLEMS IN TIME FOR WAVE EQUATIONS ON RN
}

\author{
M.W. SMILEY and A.M. FINK \\ Department of Mathematics \\ Iowa State University \\ Ames, Iowa 50011 U.S.A. \\ (Received December 5, 1989)
}

\begin{abstract}
Let $L_{\lambda}$ denote the linear operator associated with the radially symmetric form of the wave operator $\partial_{t}^{2}-\Delta+\lambda$ together with the side conditions of decay to zero as $r=\|x\| \rightarrow+\infty$ and $T$-periodicity in time. Thus $L_{\lambda} w=w_{t t}-\left(w_{r r}+\frac{N-1}{r} w_{r}\right)+\lambda w$, when there are $N$ space variables. For $\delta, R, T>0$ let $D_{T, R}=(0, T) \times(R,+\infty)$ and $L_{\delta}^{2}(D)$ denote the weighted $L^{2}$ space with weight function $\exp (\delta r)$. It is shown that $L_{\lambda}$ is a Fredholm operator from $\operatorname{dom}\left(L_{\lambda}\right) \subset L^{2}(D)$ onto $L_{\delta}^{2}(D)$ with non-negative index depending on $\lambda$. If $[2 \pi j / T]^{2}<\lambda \leq[2 \pi(j+1) / T]^{2}$ then the index is $2 j+1$. In addition it is shown that $L_{\lambda}$ has a bounded partial inverse $K_{\lambda}: L_{\delta}^{2}(D) \rightarrow$ $H_{\delta}^{1}(D) \cap L_{\delta}^{\infty}(D)$, with all spaces weighted by the function $\exp (\delta r)$. This provides a key ingredient for the analysis of nonlinear problems via the method of alternative problems.
\end{abstract}

KEY WORDS AND PHRASES. Wave equation, radial symmetry, boundary value problem, eigenvalue problem, Hilbert space, weighted Sobolev space, Fredholm operator, Laplace transform, Bessel functions.

1980 AMS SUBJECT CLASSIFICATION CODE. 35B10,35G15,35L05

\section{PROBLEM STATEMENT AND NOTATION.}

In this paper we are interested in discussing the existence of solutions for certain types of boundary value problems in time associated with linear wave equations on spatial domains of the form $\|x\|>R$, with $\|x\|=\left(\sum x_{i}^{2}\right)^{1 / 2}$. In dimensions $N=1,2$ or 3 we will allow $R=0$. One of our primary goals is the development of a linear theory that can be used in studies of nonlinear problems of the same type (cf. [1]). The most prominent case of interest is that of time-periodic solutions. Although this case is of natural interest, it has only been in the last ten years that contributions towards an understanding of this problem have been made. Many of the results on the nonlinear problem have shown nonexistence of solutions $[2],[3],[4],[5]$. These works are complemented by the existence results [6],[7] when $N=1$, and [8] when $N=3$. In this work we will extend the linear theory of [9], which played a crucial role in the analysis of [8], to all dimensions $N$ and thus set aside the restriction of $N=1$ or 3 .

Let $\Omega_{R}=\left\{x \in \mathbf{R}^{N}:\|x\|>R\right\}$. For a given real number $\lambda \in \mathbf{R}$ we will be interested in the set of solutions of the problem

$$
\begin{array}{ll}
u_{t t}-\Delta u+\lambda u=f, & (t, x) \in(0, T) \times \Omega_{R} \\
u(t, x) \rightarrow 0 \text { as }\|x\| \rightarrow+\infty, & t \in(0, T)
\end{array}
$$

which also satisfy certain boundary conditions in time. In particular these will be either a periodic condition (1.3a), or the separated boundary conditions (1.3b) (with $\alpha_{1}^{2}+\alpha_{2}^{2} \neq 0, \beta_{1}^{2}+\beta_{2}^{2} \neq 0$ all nonnegative constants) below 


$$
\begin{aligned}
& u(t+T, x)=u(t, x), \quad(t, x) \in \mathbf{R} \times \Omega_{R}, \\
& \left\{\begin{array}{l}
\alpha_{1} u(0, x)-\alpha_{2} u_{t}(0, x)=0, \\
\beta_{1} u(T, x)+\beta_{2} u_{t}(T, x)=0,
\end{array} \quad x \in \Omega_{R}\right.
\end{aligned}
$$

In the case of the periodic problem (1.1)-(1.2) should be satisfied for $t \in \mathbf{R}$.

In (1.1) the function $f \in L^{2}\left((0, T) \times \Omega_{R}\right)$ is assumed to be radially symmetric in space, so that $f(t, x)=F(t, r)$ where $r=\|x\|$. We seek solutions $u(t, x)=U(t, r)$ also having this symmetry. It is well-known in this case that $\Delta u=U_{r r}+\frac{N-1}{r} U_{r}$. Thus the standard changes of variables $w(t, r)=r^{\frac{N-1}{2}} u(t, x), h(t, r)=r^{\frac{N-1}{2}} f(t, x)$ transforms (1.1) into

$$
w_{t t}-w_{r r}+\left[\lambda+\frac{(N-1)(N-3)}{4 r^{2}}\right] w=h, \quad(t, r) \in(0, T) \times(R,+\infty)
$$

Since solutions are to be at least square-integrable we observe that $w, h \in L^{2}((0, T) \times$ $(R,+\infty))$ if and only if $u, f \in L^{2}\left((0, T) \times \Omega_{R}\right)$. In fact the solutions we find will be weak solutions in the sense described below. In particular the decay condition (1.2) will be interpreted as

$$
w(t, \cdot) \in L^{2}(R,+\infty), \quad \text { (a.e.) } t \in(0, T)
$$

Despite the apparant weakness of this decay condition it will turn out that in fact $w(t, r)$ decays exponentially to zero. In the case of the periodic conditions we can write (1.3a) in the form

$$
w(t+T, r)=w(t, r), \quad(\text { a.e. }) \quad(t, r) \in \mathbf{R} \times(R,+\infty)
$$

while the separated conditions which become

$$
\left\{\begin{array}{l}
\alpha_{1} w(0, r)-\alpha_{2} w_{t}(0, r)=0 \\
\beta_{1} w(T, r)+\beta_{2} w_{t}(T, r)=0,
\end{array} \quad r \in(R,+\infty)\right.
$$

have only a formal meaning.

Our main result can be briefly stated as follows. Let $L_{\lambda}$ be the (unbounded) linear operator associated with the boundary value problem (1.4)-(1.6). Then $L_{\lambda}$ is a Fredholm operator (cf. [10]) with finite non-negative index depending on $\lambda$. A description of the dependence of the index on $\lambda$, as well as the spaces involved, requires more notation.

For $R \geq 0$ let $D$ denote either $(R,+\infty)$ or $(0, T) \times(R,+\infty)$. Let $\delta \geq 0$ and define $L_{\delta}^{2}(D)$ to be weighted Hilbert space of function $h \in L^{2}(D)$ for which $h \cdot \exp (\delta r)$ is square-integrable over $D$. For an integer $m \geq 0$ we set (cf. [11]) $H_{\delta}^{m}(D)=\left\{h \in L_{\delta}^{2}(D): D^{\alpha} h \in L_{\delta}^{2}(D)\right.$ for $0 \leq|\alpha| \leq m\}$. The derivatives are to be understood as distributional derivatives relative to $C_{0}^{\infty}(R,+\infty)$ or $\mathcal{D}_{T, R}$ as defined below, respectively. The norm used in $L_{\delta}^{2}(D)$ will be

$$
\|h\|_{L_{6}^{2}(D)}=\left\{\int_{D}|h|^{2} e^{2 \delta r} d \mu\right\}^{1 / 2}, \quad(d \mu=d r \quad \text { or } \quad d r d t \quad \text { resp. })
$$

and in $H_{\delta}^{m}(D)$ we use

$$
\|h\|_{H_{6}^{m}(D)}=\left\{\sum_{|\alpha| \leq m}\left\|D^{\alpha} h\right\|_{L_{\delta}^{2}(D)}^{2}\right\}^{1 / 2}
$$


Similarly we define $L_{\delta}^{m, \infty}(D)$ as the set of functions $h \in L^{\infty}(D)$ for which $\left|D^{\alpha} h\right| \exp (\delta r)$ is bounded on $D$, for all $0 \leq|\alpha| \leq m$. When $m=0$ we more briefly write $L_{\delta}^{\infty}(D)$.

The class of test functions to be used in defining solutions of (1.4)-(1.6) is $\mathcal{D}_{T, R}=\left\{\varphi \in C^{\infty}(\mathbf{R} \times(R,+\infty)): \varphi\right.$ satisfies $(1.6)$ and $\left.\varphi(t, \cdot) \in C_{0}^{\infty}(R,+\infty)\right\}$. Let $q_{N}(r)=$ $(N-1)(N-3) / 4 r^{2}$. We say that $w \in L^{2}((0, T) \times(R,+\infty))$ is a weak solution of (1.4)-(1.6) if

$$
\int_{0}^{T} \int_{R}^{+\infty} w\left[\varphi_{t t}-\varphi_{r r}+\left(\lambda+q_{N}(r)\right) \varphi\right] d r d t=\int_{0}^{T} \int_{R}^{+\infty} h \varphi d r d t, \quad \forall \varphi \in \mathcal{D}_{T, R} .
$$

The operator $L_{\lambda}$ defined formally above is now to be understood as a closed linear operator in $L^{2}((0, T) \times(R,+\infty))$, with $\operatorname{dom}\left(L_{\lambda}\right) \subset H_{\delta}^{1}((0, T) \times(R,+\infty))$

The distributional definition above (cf. [12]) allows for an analysis based on separation of variables. It is well known (cf. [13]) that the eigenvalue problems

$$
\begin{aligned}
& \xi^{\prime \prime}+\theta^{2} \xi=0, \quad 0<t<T, \\
& \xi(0)=\xi(T), \quad \xi^{\prime}(0)=\xi^{\prime}(T), \quad \text { or } \\
& \alpha_{1} \xi(0)-\alpha_{2} \xi^{\prime}(0)=0, \quad \beta_{1} \xi(T)+\beta_{2} \xi^{\prime}(T)=0
\end{aligned}
$$

admit countably infinite sequences of eigenvalues $\left\{\theta_{n}^{2}\right\}$ and eigenfunctions $\left\{\xi_{n}(t)\right\}$. The eigenfunctions are known to be complete in the sense that linear combinations are dense in $L^{2}(0, T)$. Allowing for the different cases in (1.9) we assume $\left\{\theta_{n}\right\}$ is enumerated so that the sequence is nondecreasing and includes repetition in the periodic case. In terms of these notations our main result can be stated as follows.

THEOREM. If $\theta_{j}^{2}<\lambda \leq \theta_{j+1}^{2}$ for some $j$ and $0<\delta<\left(\lambda-\theta_{j}^{2}\right)^{1 / 2}$ then the operator $L_{\lambda}$ is a Fredholm operator onto $L_{\delta}^{2}((0, T) \times(R,+\infty))$ with index equal to the dimension of the linear space spanned by $\left\{\xi_{n}(t)\right\}_{n \leq \jmath}$. Moreover, if the boundary conditions (1.9) are either periodic, Dirichlet, or Neumann, then there is a bounded partial inverse $K_{\lambda}: L_{\delta}^{2}((0, T) \times(R,+\infty)) \rightarrow$ $H_{\delta}^{1}((0, T) \times(R,+\infty))$ such that $L_{\lambda} w=h$ if and only if $w=v+K_{\lambda} h$, where $v \in \operatorname{ker}\left(L_{\lambda}\right)$. In addition range $\left(K_{\lambda}\right) \subset \operatorname{ker}\left(L_{\lambda}\right)^{\perp}$ with the orthogonality in $H_{\delta}^{1}((0, T) \times(R,+\infty))$.

We loosely describe the arguments leading to the above result. Let $\varphi(t, r)=\xi_{n}(t) \psi(r)$ for some $\psi \in C_{0}^{\infty}(R,+\infty)$. Then (1.7) implies

$$
\int_{R}^{+\infty} w_{n}\left[-\psi^{\prime \prime}+\left(-\theta_{n}^{2}+\lambda+q_{N}(r)\right) \psi\right] d r=\int_{R}^{+\infty} h_{n} \psi d r
$$

where

$$
w_{n}(r)=c_{n}^{-1} \int_{0}^{T} w(r, s) \xi_{n}(t) d t, \quad h_{n}(r)=c_{n}^{-1} \int_{0}^{T} w(t, r) \xi_{n}(t) d t
$$

for a constant $c_{n}=\left\|\xi_{n}\right\|_{L^{2}(0, T)}^{2}$. Thus it follows that $w_{n}(r)$ is a solution of the generalized boundary value problem

$$
\begin{aligned}
w_{n}^{\prime \prime}+p_{n}(r) w_{n} & =-h_{n}, \quad r \in(R,+\infty), \\
w_{n} & \in L^{2}(R,+\infty)
\end{aligned}
$$


where $p_{n}(r)=\theta_{n}^{2}-\lambda-(N-1)(N-3) / 4 r^{2}$. On the other hand, if solutions of these problems can be found for every $n$ then $w(t, r) \sim \sum w_{n}(r) \xi_{n}(t)$ should satisfy (1.4)-(1.6) in the sense of (1.7). In this way we may show existence of solutions. However we are interested in uniqueness and continuous dependence as well as existence of solutions. Thus we must characterize all solutions of (1.12)-(1.13). In particular we need to show (1.13) is a property which guarantees uniqueness. When $\theta_{n}^{2} \geq \lambda$ this can be done. When $\theta_{n}^{2}<\lambda$ an additional constraint is needed to obtain a particular 1-dimensional affine space of solutions. Verification of these remarks requires several steps.

First we show existence and uniqueness of solutions to a more restrictive problem in which (1.13) is replaced by the requirement $w \in L_{\delta}^{2}(R,+\infty)$ for some $\delta>0$. At the same time, a priori estimates of solutions are given in the $L_{\delta}^{2}(R,+\infty)$ norm. These estimates are crucial for subsequent developments, and are stronger than would be suggested by (1.13). To bridge the gap between the competing desires for strong estimates on one hand and weak uniqueness conditions on the other hand we consider an intermediate problem in which (1.13) is replaced by $w \in L_{\delta-\varepsilon}^{2}(R,+\infty)$ for some $\varepsilon, 0 \leq \varepsilon \leq \delta$. We then show a priori estimates can be obtained when $\varepsilon=0$, and that uniqueness is implied when $\varepsilon=\delta$.

The outline of the arguments for this are as follows. In the next section existence and uniqueness is established with $\varepsilon<\delta$. Some a priori estimates, which require $R$ large, are also given. Then in the third section some special solutions are considered. These solutions are shown to satisfy a priori estimates with arbitrary $R$ but require $\varepsilon>0$. Finally in Section 4 the results of the previous sections are combined to obtain a characterization of solutions and a priori estimates.

Throughout the developments we have outlined, it is essential to show that the constants involved in the a priori estimates are independent of $n$. This allows for a passage to the limit argument. This argument is given in Section 5 and is used to show that solutions of (1.4)-(1.6) exist as limits of sums of the component functions described above.

\section{A CHARACTERIZATION OF SOLUTIONS.}

It is crucial for our purposes that we completely characterize solutions of the generalized boundary value problems (1.12)-(1.13). Towards this goal we consider the problem

$$
\begin{gathered}
w^{\prime \prime}+p(r) w=h, \quad r \in(R,+\infty), \\
w \in L_{\delta-\varepsilon}^{2}(R,+\infty),
\end{gathered}
$$

where $p(r)=b-q(r)$ with $b \in \mathbf{R}$ and $q(r)=r^{-2}\left(\sigma^{2}-\frac{1}{4}\right)$. In particular we want to include the case $b=b_{n}=\theta_{n}^{2}-\lambda \geq-\lambda$ (i.e. bounded below) and $\sigma=(N-2) / 2$. With these choices $p=p_{n}$ and $q=q_{N}$ are the functions of the previous section. In general we assume $\delta>0$ and if $b<0$ then also $\delta<|b|^{1 / 2}, R \geq 0,0 \leq \varepsilon \leq \delta$, and $h \in L_{\delta}^{2}(R,+\infty)$. When $N=1$ or 3 so that $p(r)=b$ is constant, and with $R=0$, an analysis of problem (2.1)-(2.2) has been given in [9]. The analysis uses the classical Paley-Weiner Theorem [14] (cf. [15]) which characterizes functions $h \in L^{2}(0,+\infty)$ in terms of their Laplace transforms $H(s)=\mathcal{L}\{h(r)\}$. We summarize a number of the results obtained in [9] in the statement following.

THEOREM 2.1. Let $\beta$ denote a non-negative real number, and let $p(r)=b, R=0$, and $\varepsilon=\delta$ in $(2.1)-(2.2)$. 
i) If $b=\beta^{2} \geq 0$ and $0<\delta<+\infty$ then, for every $h \in L_{\delta}^{2}(0,+\infty)$, there is a unique solution $\tilde{w}$ of (2.1)-(2.2).

ii) If $b=-\beta^{2}<0$ and $0<\delta<\beta$ then, for every $h \in L_{\delta}^{2}(0,+\infty)$, (2.1)-(2.2) has a 1dimensional affine space of solutions. All solutions have the form

$w(r)=\alpha \exp (-\beta r)+\tilde{w}(r)$ where $\alpha \in \mathbf{R}$ is arbitrary and

$\widetilde{w}(r)=\mathcal{L}^{-1}\left\{[H(s)-H(\beta)] /\left(s^{2}-\beta^{2}\right)\right\}$ is uniquely determined by $h$.

Furthermore the function $\tilde{w}$, in either case, belongs to $H_{\delta}^{2}(0,+\infty)$ and satisfies the a priori estimates

$$
\begin{aligned}
& \|\tilde{w}\|_{L_{\delta}^{2}(0,+\infty)} \leq C\|h\|_{L_{\delta}^{2}(0,+\infty)}, \quad|b|^{1 / 2}\|\tilde{w}\|_{L_{\delta}^{2}(0,+\infty)} \leq C\|h\|_{L_{\delta}^{2}(0,+\infty)}, \\
& \|\tilde{w}\|_{L_{6}^{1, \infty}(0,+\infty)} \leq C\|h\|_{L_{6}^{2}(0,+\infty)},
\end{aligned}
$$

where $C=C(\delta)$ if $b \geq 0$, or $C=C(\delta, \beta-\delta)$ if $b<0$, is a constant independent of $h$ and such that $C \rightarrow+\infty$ as $\delta \rightarrow 0^{+}$, and as $\delta \rightarrow \beta^{-}$if $b<0$.

REMARK: The above result shows that uniqueness is obtained by only requiring $w \in$ $L^{2}(0,+\infty)$, and that the uniquely determined solutions actually belong to $L_{\delta}^{2}(0,+\infty)$ where $\delta>0$ is such that $h \in L_{\delta}^{2}(0,+\infty)$. If we only know that $h \in L^{2}(0,+\infty)$ then there is no guarantee that solutions exist (cf. [9]).

Below we need to consider (2.1)-(2.2) where generally we must have $R>0$. We will comment on the exceptional cases $R \geq 0$ subsequently. If $0<\delta<\beta$ then $\exp (-\beta r) \in L_{\delta}^{2}(R,+\infty)$ for any $R \geq 0$. Let $\mathcal{N}_{R}$ denote the 1-dimensional subspace of $L_{\delta}^{2}(R,+\infty)$ spanned by $\exp (-\beta r)$, and let

$$
Q: L_{\delta}^{2}(R,+\infty) \rightarrow \mathcal{N}_{R}
$$

denote the orthogonal projection onto $\mathcal{N}_{R}$. An easy calculation shows $Q h(r)=c \exp (-\beta r)$, where $c=2(\beta-\delta) \exp (\beta R) H_{R}(\beta-2 \delta)$ with $H_{R}(s)=\mathcal{L}\{h(r+R)\}$. Clearly we have the orthogonal decomposition $L_{\delta}^{2}(R,+\infty)=\mathcal{N}_{R}+\mathcal{N}_{R}^{\perp}$, with $Q w=0$ for all $w \in \mathcal{N}_{R}^{\perp}$.

COROLLARY 2.2. Let $\beta$ denote a non-negative real number, and let $p(r)=b, R \geq 0$, and $\varepsilon=\delta$ in (2.1)-(2.2). Then there is a continuous linear solution operator $\mathcal{K}_{b}$ in $L_{\delta}^{2}(R,+\infty)$ for (2.1)-(2.2). $\mathcal{K}_{b}$ is defined according to the following cases:

i) If $b=\beta^{2} \geq 0$ and $0<\delta<+\infty$ then $\mathcal{K}_{b}: L_{\delta}^{2}(R,+\infty) \rightarrow L_{\delta}^{2}(R,+\infty)$ is defined by $\mathcal{K}_{b} h=\tilde{w}$ where $\tilde{w}$ is the unique solution of the problem. Thus $w$ satisfies (2.1)-(2.2) if and only if $w=\tilde{w}=\mathcal{K}_{b} h$.

ii) If $b=-\beta^{2}<0$ and $0<\delta<\beta$ then $\mathcal{K}_{b}: L_{\delta}^{2}(R,+\infty) \rightarrow \mathcal{N}_{R}^{1}$ is defined by $\mathcal{K}_{b} h=\widetilde{\widetilde{w}}$ where $\widetilde{\widetilde{w}}$ is the unique solution of (2.1)-(2.2) satisfying $Q \widetilde{\widetilde{w}}=0$. Thus $w$ satisfies (2.1)-(2.2) if and only if $w(r)=\alpha \exp (-b r)+\widetilde{\widetilde{w}}(r)$ where $\alpha \in \mathbf{R}$ and $\tilde{\widetilde{w}}=\mathcal{K}_{b} h$. 
Furthermore we have in either case $\mathcal{K}_{b} h \in L_{\delta}^{1, \infty}(R,+\infty) \cap H_{\delta}^{2}(R,+\infty)$ and

$$
\begin{aligned}
& \left\|\mathcal{K}_{b} h\right\|_{L_{6}^{2}(R,+\infty)} \leq C\|h\|_{L_{6}^{2}(R,+\infty)}, \quad|b|^{1 / 2}\left\|\mathcal{K}_{b} h\right\|_{L_{6}^{2}(R,+\infty)} \leq C\|h\|_{L_{6}^{2}(R,+\infty)} \\
& \left\|\mathcal{K}_{b} h\right\|_{L_{6}^{1, \infty}(R,+\infty)} \leq C\|h\|_{L_{6}^{2}(R,+\infty)},
\end{aligned}
$$

for a constant $C$ having the same character as in (2.3)-(2.4). In particular there is a constant $K_{0}$ independent of $R$ such that $\left\|\mathcal{K}_{b}\right\| \leq K_{0}$ with the norm being the linear operator norm in $L_{\delta}^{2}(R,+\infty)$.

PROOF: Given a function $w$ defined on $(R,+\infty)$ we set $w_{R}(r)=w(r+R)$ to obtain the translate defined on $(0,+\infty)$. If $w \in L_{\delta}^{2}(R,+\infty) \cap L_{\delta}^{\infty}(R,+\infty)$ then

$$
\left\|w_{R}\right\|_{L_{\delta}^{2}(0,+\infty)}=e^{-\delta R}\|w\|_{L_{\delta}^{2}(R,+\infty)}, \quad\left\|w_{R}\right\|_{L_{\delta}^{\infty}(0,+\infty)}=e^{-\delta R}\|w\|_{L_{\delta}^{\infty}(R,+\infty)} .
$$

Suppose that $w$ satisfies (2.1)-(2.2). Then $w_{R}$ satisfies (2.1)-(2.2) on $(0,+\infty)$ with $h$ replaced by $h_{R}$. Hence either $w_{R}=\tilde{w}_{R}$ is uniquely determined $\left(b=\beta^{2} \geq 0\right)$, or has the form $w_{R}(r)=$ $\alpha \exp (-\beta r)+\tilde{w}_{R}(r)$ as described in Theorem 2.1. It follows that the same is true for $w(r)=$ $w_{R}(r-R)$. Let $\tilde{w}(r)=\tilde{w}_{R}(r-R)$. Then by $(2.3)$ and $(2.8)$

$$
\|\tilde{w}\|_{L_{6}^{2}(R,+\infty)}=e^{\delta R}\left\|\tilde{w}_{R}\right\|_{L_{6}^{2}(0,+\infty)} \leq C e^{\delta R}\left\|h_{R}\right\|_{L_{6}^{2}(0,+\infty)}=C\|h\|_{L_{6}^{2}(R,+\infty)} .
$$

Repeating this argument using the other norms involved shows that $\tilde{w}$ satisfies the estimates (2.3)-(2.4) on $(R,+\infty)$, and when $b \geq 0$ this verifies (2.6)-(2.7). If $b<0$ then we need to show the same estimates apply for $\tilde{\widetilde{w}}=(I-Q) \tilde{w}$. But since $Q$ is an orthogonal projection in $L_{\delta}^{2}(R,+\infty)$ (2.6) follows immediately. To obtain (2.7) we first observe that if $c \exp (-\beta r)=Q \tilde{w}(r)$ then again by orthogonality

$$
|c| \frac{e^{-(\beta-\delta) R}}{\sqrt{2(\beta-\delta)}}=\left\|c e^{-\beta(\cdot)}\right\|_{L_{6}^{2}(R,+\infty)} \leq\|\tilde{w}\|_{L_{6}^{2}(R,+\infty)} .
$$

Hence by the triangle inequality

$$
\begin{aligned}
& e^{\delta r}|\tilde{\widetilde{w}}(r)| \leq e^{\delta r}|\tilde{w}(r)|+\sqrt{2(\beta-\delta)} e^{-(\beta-\delta)(r-R)}\|\tilde{w}\|_{L_{\delta}^{2}(R,+\infty)}, \quad r>R, \\
& e^{\delta r}\left|\tilde{\widetilde{w}}^{\prime}(r)\right| \leq e^{\delta r}\left|\tilde{w}^{\prime}(r)\right|+\sqrt{2(\beta-\delta)} e^{-(\beta-\delta)(r-R)}|b|^{1 / 2}\|\tilde{w}\|_{L_{\delta}^{2}(R,+\infty)}, \quad r>R .
\end{aligned}
$$

(2.7) now follows from these inequalities and the estimates already established for $\tilde{w}$.

We now consider the variable coefficient problem originally stated. Assuming $q(r) \rightarrow 0$ as $r \rightarrow+\infty$ one might conjecture that this problem will admit unique or multiple solutions, according to the value of $b$, in the same way as described in Theorem 2.1. That is the problems should be asymptotically equivalent in the sense of solution sets.

THEOREM 2.3. Let $\beta$ denote a non-negative real number, and let $0 \leq \varepsilon<\delta$ in (2.2). Let $p(r)=b-q(r)$ in (2.1) with $b \in \mathbf{R}$ and $q(r)=\left(\sigma^{2}-\frac{1}{4}\right) / r^{2}$. Then there is a number $R_{0}>0$ such that when $R=R_{0}$ in (2.1)-(2.2) the following holds:

i) If $b=\beta^{2} \geq 0$ and $0<\delta<+\infty$ then, for every $h \in L_{\delta}^{2}\left(R_{0},+\infty\right)$, there is a unique solution $\widehat{w}$ of $(2.1)-(2.2)$. 
ii) If $b=-\beta^{2}<0$ and $0<\delta<\beta$ then, for every $h \in L_{\delta}^{2}\left(R_{0},+\infty\right)$, problem (2.1)-(2.2) has a 1-dimensional affine space of solutions. Moreover there is a solution $\widehat{w}$ of (2.1)-(2.2) such that all solutions have the form $w(r)=\alpha w_{0}(r)+\widehat{w}(r)$ where $\alpha \in \mathbf{R}$ and $w_{0}(r)$ is a solution of the corresponding homogeneous problem which satisfies (2.2).

In either case the solution $\widehat{w}$ satisfies the following estimates for a constant $C$ having the same dependence on $\delta$, and $\beta-\delta$ if $b<0$, as in (2.3)-(2.4).

$$
\begin{aligned}
& \|\widehat{w}\|_{L_{6}^{2}\left(R_{0},+\infty\right)} \leq C\|h\|_{h_{6}^{2}\left(R_{0},+\infty\right)}, \quad|b|^{1 / 2}\|\widehat{w}\|_{L_{6}^{2}\left(R_{0},+\infty\right)} \leq C\|h\|_{L_{6}^{2}\left(R_{0},+\infty\right)}, \\
& \|\widehat{w}\|_{L_{6}^{1, \infty}\left(R_{0},+\infty\right)} \leq C\|h\|_{L_{6}^{2}\left(R_{0},+\infty\right)} .
\end{aligned}
$$

PROOF: First we assume $\varepsilon=0$. For convenience set $c_{0}=\left|\sigma^{2}-\frac{1}{4}\right|$. Let $\mathcal{K}_{b}$ be the linear operator defined in Corollary 2.2, and suppose $b=\beta^{2} \geq 0$. Let $K_{0}$ denote a bound for the operator norm of $\mathcal{K}_{b}$ in $L_{\delta}^{2}(R,+\infty)$. We can assume $K_{0}$ is independent of $R$. Choose $R_{0}>\left(K_{0} c_{0}\right)^{1 / 2}$. By the corollary we know $w \in L_{\delta}^{2}\left(R_{0},+\infty\right)$ satisfies (2.1)-(2.2) if and only if $w=\mathcal{K}_{b}[q w+h]$. Let $T: L_{\delta}^{2}\left(R_{0},+\infty\right) \rightarrow L_{\delta}^{2}\left(R_{0},+\infty\right)$ be the bounded affine operator defined by $T w=\mathcal{K}_{b}[q w+h]$. We have

$$
\begin{aligned}
\|T w-T v\|_{L_{6}^{2}\left(R_{0},+\infty\right)} & \leq\left\|\mathcal{K}_{b}\right\|\|q\|_{L^{\infty}\left(R_{0},+\infty\right)}\|w-v\|_{L_{\delta}^{2}\left(R_{0},+\infty\right)} \\
& \leq K_{0} c_{0} R_{0}^{-2}\|w-v\|_{L_{6}^{2}\left(R_{0},+\infty\right)}
\end{aligned}
$$

It follows that $T$ is a contraction by the choice of $R_{0}$. Hence there is a uniquely determined fixed point $\widehat{w} \in L_{\delta}^{2}\left(R_{0},+\infty\right)$. Equivalently, when $\varepsilon=0$ and $R=R_{0}$, problem (2.1)-(2.2) has a unique solution $\widehat{w} \in L_{\hat{6}}^{2}\left(R_{0},+\infty\right)$.

If $b=-\beta^{2}<0$ then $w \in L_{\delta}^{2}\left(R_{0},+\infty\right)$ is a solution of (2.1)-(2.2) if and only if $w(r)=$ $\alpha \exp (-\beta r)+\tilde{\widetilde{w}}(r)$, where $\widetilde{\tilde{w}}=\mathcal{K}_{b}[q w+h]$. Now for a fixed $\alpha \in \mathbf{R}$, define $T: \mathcal{N}_{R_{0}}^{\perp} \rightarrow \mathcal{N}_{R_{0}}^{\perp}$ by $T w_{1}=\mathcal{K}_{b}[q w+h]$, where $w(r)=\alpha \exp (-\beta r)+w_{1}(r)$. Then as before we find

$$
\left\|T w_{1}-T v_{1}\right\|_{L_{6}^{2}\left(R_{0},+\infty\right)} \leq K_{0} c_{0} R_{0}^{-2}\left\|w_{1}-v_{1}\right\|_{L_{6}^{2}\left(R_{0},+\infty\right)}
$$

for all $w_{1}, v_{1} \in \mathcal{N}_{R_{0}}^{\perp}$. Therefore $T$ has a unique fixed point $w_{1} \in \mathcal{N}_{R_{0}}^{\perp}$, and all solutions of (2.1)-(2.2) belonging to $L_{\delta}^{2}\left(R_{0},+\infty\right)$ have the form $w(r)=\alpha \exp (-\beta r)+w_{1}(r)$.

For convenience of notation let $e(r)=\exp (-\beta r)$. Since the operator $\mathcal{K}_{b} q$ has norm less than one it follows that $w_{1}=\left(I-\mathcal{K}_{b} q\right)^{-1} \mathcal{K}_{b}[q \alpha e+h]$. Thus $w_{1}$ depends linearly and continuously on both $\alpha \in \mathbf{R}$ and $h \in L_{\delta}^{2}\left(R_{0},+\infty\right)$. In particular we may write $w_{1}(r)=\alpha w_{2}(r)+\widehat{w}(r)$, where

$$
w_{2}=\left(I-\mathcal{K}_{b} q\right)^{-1} \mathcal{K}_{b} q e, \quad \widehat{w}=\left(I-\mathcal{K}_{b} q\right)^{-1} \mathcal{K}_{b} h
$$

Hence $w(r)=\alpha\left(\exp (-\beta r)+w_{2}(r)\right)+\widehat{w}(r)$, where clearly $w_{0}(r)=\exp (-\beta r)+w_{2}(r)$ is a solution of the corresponding homogeneous problem which satisfies $w_{0} \in L_{\delta}^{2}\left(R_{0},+\infty\right)$, and $\widehat{w}$ is a solution of $(2.1)$ which is uniquely determined by $h$.

It remains to verify the estimates (2.9)-(2.10). First assume $b \geq 0$. Then the bound $K_{0}$ is also independent of $b$. Hence both $\mathcal{K}_{b}$ and $\left(I-\mathcal{K}_{b} q\right)^{-1}$ are norm bounded independent of $b$ as operators in $L_{\delta}^{2}\left(R_{0},+\infty\right)$. Therefore $\widehat{w}=\left(I-\mathcal{K}_{b} q\right)^{-1} \mathcal{K}_{b} h$ satisfies the first estimate in (2.9). Using this estimate in conjunction with (2.6)-(2.7), with $h$ replaced by $q \widehat{w}+h$, yields (2.9)-(2.10). Now 
suppose $b<0$. Then the bound $K_{0}$ depends on the difference $\beta-\delta$. It follows that $\left\|\left(I-\mathcal{K}_{b} q\right)^{-1}\right\|$ inherits this dependence. As before $\widehat{w}$, as given by (2.11), satisfies the first inequality in (2.9) with the constant $C$ now depending on $\beta-\delta$. Since $\widehat{w}=\mathcal{K}_{b}[q \widehat{w}+h]$ we can use the same boot-strap argument as above to obtain the remaining estimates in (2.9)-(2.10).

Now suppose $0<\varepsilon<\delta$. Then $\tilde{\delta}=\delta-\varepsilon>0$ and $h \in L_{\delta}^{2}\left(R_{0},+\infty\right) \subset L_{\tilde{\delta}}^{2}\left(R_{0},+\infty\right)$. Hence by the argument just given, with $\tilde{\delta}$ replacing $\delta$, there is a unique solution of (2.1) satisfying $w \in L_{\tilde{\delta}}^{2}(R,+\infty)$, when $b \geq 0$ and $R \geq R_{0}$. But $\widehat{w} \in L_{\tilde{\delta}}^{2}\left(R_{0},+\infty\right)$ and therefore $w=\widehat{w}$ in this case. If $b<0$ only the existence of $\widehat{w}$ has been claimed. The characterization of solutions follows as before.

REMARK: The argument above fails when $\varepsilon=\delta$. In this case we would have $\tilde{\delta}=0$ and hence $\mathcal{K}_{b}$ would not be well-defined.

COROLLARY 2.4. Let $p(r)=b-q(r)$ where $b \in \mathbf{R}$ and $q(r)=\left(\sigma^{2}-\frac{1}{4}\right) / r^{2}$. Assume $0 \leq \varepsilon<\delta$. Then conclusions i) and ii) of the previous theorem are valid with $R_{0}$ replaced by any $R>0$. In addition estimates (2.9)-(2.10) remain valid for $R_{0}$ sufficiently large.

PROOF: This follows immediately by continuation of the unique solution $\widehat{w}$ to $(0,+\infty)$, which is possible since $p \in C(0,+\infty)$.

\section{SPECIAL SOLUTIONS.}

In this section we consider some special solutions obtained from the variation of parameters formula by certain choices of the free constants. Letting $p(r)=b-q(r)$, where $b \in \mathbf{R}$ and $q(r)=\left(\sigma^{2}-\frac{1}{4}\right) / r^{2}$ with $\sigma \geq-\frac{1}{2}$, differential equation (2.1) becomes

$$
w^{\prime \prime}+\left(b-\frac{\sigma^{2}-\frac{1}{4}}{r^{2}}\right) w=h
$$

This is a nonhomogeneous Bessel equation. All solutions have the form

$$
w(r)=\left(c_{1}-\int_{R}^{r} \frac{w_{2}(s) h(s)}{W(s)} d s\right) w_{1}(r)+\left(c_{2}+\int_{R}^{r} \frac{w_{1}(s) h(s)}{W(s)} d s\right) w_{2}(r),
$$

in which $c_{1}, c_{2}$ are arbitrary constants, $w_{1}(r)$ and $w_{2}(r)$ are linearly independent solutions of the corresponding homogeneous problem, and $W(s)$ is their Wronskian. It follows immediately from Abel's formula that $W(s)$ is constant.

There are three distinct cases to consider. Let $\nu=|\sigma|$. If $b=\beta^{2}$ for some $\beta>0$ then we may take (cf. [16],[17]) $w_{1}(r)=r^{1 / 2} J_{\nu}(\beta r)$ and $w_{2}(r)=r^{1 / 2} Y_{\nu}(\beta r)$ where $J_{\nu}, Y_{\nu}$ denote Bessel functions of the 1st and 2 nd kind respectively. From known results (cf. [16]) it follows that $W(s)=\frac{2}{\pi}$. Since we know the unique solution $\widehat{w}$ of (2.1)-(2.2) tends to zero as $r \rightarrow+\infty$, while $w_{1}(r)$ and $w_{2}(r)$ are asymptotically cosine and sine functions, we hueristically argue that the choices

$$
c_{1}=\frac{\pi}{2} \int_{R}^{+\infty} s^{1 / 2} Y_{\nu}(\beta s) h(s) d s, \quad c_{2}=-\frac{\pi}{2} \int_{R}^{+\infty} s^{1 / 2} J_{\nu}(\beta s) h(s) d s
$$

should yield $\widehat{w}$. With these choices $(3.2)$ becomes

$$
\begin{aligned}
& w(r)=\int_{r}^{+\infty} G_{b, \nu}(r, s) h(s) d s, \quad \text { where } \\
& G_{b, \nu}(r, s)=\frac{\pi}{2}(r s)^{1 / 2}\left[J_{\nu}(\beta r) Y_{\nu}(\beta s)-J_{\nu}(\beta s) Y_{\nu}(\beta r)\right] .
\end{aligned}
$$


If $b=0$ then we can take $w_{1}(r)=r^{\frac{1}{2}+\nu}, w_{2}(r)=r^{\frac{1}{2}-\nu}$ when $\nu \neq 0$ and $w_{1}(r)=r^{1 / 2}$, $w_{2}(r)=r^{1 / 2} \log r$ when $\nu=0$. Direct calculation shows $W(s)=-2 \nu$ or $W(s)=1$ respectively. Again hueristics suggest

$$
c_{1}=\left\{\begin{array}{ll}
-\frac{1}{2 \nu} \int_{R}^{+\infty} s^{\frac{1}{2}-\nu} h(s) d s, & \nu \neq 0 \\
\int_{R}^{+\infty} s^{1 / 2} \log s h(s) d s, & \nu=0
\end{array}, \quad c_{2}= \begin{cases}\frac{1}{2 \nu} \int_{R}^{+\infty} s^{\frac{1}{2}+\nu} h(s) d s, & \nu \neq 0 \\
-\int_{R}^{+\infty} s^{1 / 2} h(s) d s, & \nu=0\end{cases}\right.
$$

so that (3.2) now takes the form (3.3) with

$$
G_{0, \nu}(r, s)=\left\{\begin{array}{l}
-\frac{1}{2 \nu}\left(r^{\frac{1}{2}+\nu} s^{\frac{1}{2}-\nu}-s^{\frac{1}{2}+\nu} r^{\frac{1}{2}-\nu}\right), \quad \nu \neq 0 \\
(r s)^{1 / 2} \log (s / r), \quad \nu=0
\end{array}\right.
$$

If $b=-\beta^{2}$ for some $\beta>0$ then we take $w_{1}(r)=r^{1 / 2} I_{\nu}(\beta r)$ and $w_{2}(r)=r^{1 / 2} K_{\nu}(\beta r)$ where $I_{\nu}, K_{\nu}$ are the modified Bessel functions. In this case $W(s)=-1$. Here we make the ansatz

$$
c_{1}=-\int_{R}^{+\infty} s^{1 / 2} K_{\nu}(\beta s) h(s) d s, \quad c_{2}=0
$$

Then (3.2) becomes

$$
\begin{aligned}
& w(r)=\int_{R}^{+\infty} G_{b, \nu}(r, s) h(s) d s, \quad \text { where } \\
& G_{b, \nu}(r, s)= \begin{cases}-(r s)^{1 / 2} I_{\nu}(\beta s) K_{\nu}(\beta r), & R<s<r, \\
-(r s)^{1 / 2} I_{\nu}(\beta r) K_{\nu}(\beta s), & r<s<+\infty .\end{cases}
\end{aligned}
$$

Ultirnately we need estimates on $\|w\|$ and $|b|^{1 / 2}\|w\|$ in the $L_{\delta-\varepsilon}^{2}(R,+\infty)$ norm for the functions $w(r)$ defined in (3.3)-(3.7). For convenience we assume that $b_{0} \geq 1$ is a fixed number. Then both $\|w\|$ and $|b|^{1 / 2}\|w\|$ are bounded by $\max \left(b_{0}^{1 / 2},|b|^{1 / 2}\right)\|w\|$. Thus it suffices to bound this last quantity. In general we have by Hölder's inequality

$$
|w(r)|^{2} \leq\|h\|_{L_{6}^{2}(R,+\infty)}^{2} \int_{R}^{+\infty}\left|G_{b, \nu}(r, s)\right|^{2} e^{-2 \delta s} d s
$$

with $G_{b, \nu}(r, s)$ extended as the zero function on $R \leq s<r$ in case $b \geq 0$. Setting $\tilde{G}_{b, \nu}(r, s)=$ $\max \left(b_{0}^{1 / 2},|b|^{1 / 2}\right) G_{b, \nu}(r, s)$ we thus obtain

$$
\begin{gathered}
\max \left(b_{0}^{1 / 2},|b|^{1 / 2}\right)\|w\|_{L_{\delta-\varepsilon}^{2}(R,+\infty)} \leq C(b, R, \delta, \varepsilon, \nu)\|h\|_{L_{\delta}^{2}(R,+\infty)}, \quad \text { assuming } \\
\left\{\int_{R}^{+\infty} \int_{R}^{+\infty}\left|\tilde{G}_{b, \nu}(r, s)\right|^{2} e^{-2[\delta(s-r)+r \varepsilon]} d s d r\right\}^{1 / 2} \leq C(b, R, \delta, \varepsilon, \nu) .
\end{gathered}
$$

Our goal is to show that the constant $C(b, R, \delta, \varepsilon, \nu)$ is finite and has the following types of dependence on the various parameters:

(3.10i) $C(b, R, \delta, \varepsilon, \nu) \rightarrow+\infty$ as $\delta \rightarrow 0^{+}$ 
(3.10ii) If $b \geq 0$ then $C(b, R, \delta, \varepsilon, \nu)=C(R, \delta, \varepsilon, \nu)$ is independent of $b$, and if $b<0$ then $C(b, R, \delta, \varepsilon, \nu)=C\left(|b|^{1 / 2}-\delta, R, \delta, \varepsilon, \nu\right)$ depends on $b$ only through the difference $|b|^{1 / 2}-$ $\delta>0$ and tends to $+\infty$ as $\delta \rightarrow|b|^{1 / 2}$.

(3.10iii) If $0 \leq \nu \leq \frac{1}{2}$ then $C(b, R, \delta, \varepsilon, \nu)=C(b, \nu, \varepsilon, \nu)$ is independent of $R$, and if $\nu>\frac{1}{2}$ then $C(b, R, \delta, \varepsilon, \nu) \rightarrow+\infty$ as $R \rightarrow 0^{+}$.

There are several cases that must be considered. First suppose that $b=\beta^{2}>0$ so that $G_{b, \nu}(r, s)$ is given by (3.4). We define two related functions $F_{\nu}, Q_{\nu}: \mathbf{R}^{+} \rightarrow \mathbf{R}$ for $\nu>0$ by the formulas

$$
F_{\nu}(x)=\left(\frac{x}{2}\right)^{-\nu} J_{\nu}(x), \quad Q_{\nu}(x)=\left(\frac{x}{2}\right)^{\nu} Y_{\nu}(x)
$$

From the well-known asymptotics (cf. [1]) for $J_{\nu}, Y_{\nu}$ it follows that $F_{\nu}(x)=\mathcal{O}(1), Q_{\nu}(x)=\mathcal{O}(1)$ as $x \rightarrow 0^{+}$and $F_{\nu}(x)=\mathcal{O}\left(x^{-\nu-\frac{1}{2}}\right), Q_{\nu}(x)=\mathcal{O}\left(x^{\nu-\frac{1}{2}}\right)$ as $x \rightarrow+\infty$. Consequently $F_{\nu} \in$ $L^{\infty}\left(\mathbf{R}^{+}\right)$and if $0<\nu \leq \frac{1}{2}$ then $Q_{\nu} \in L^{\infty}\left(\mathbf{R}^{+}\right)$. Also we have $\left(\frac{x}{2}\right)^{\nu+\frac{1}{2}} F_{\nu}(x)$ bounded on $\mathbf{R}^{+}$, and $\left(\frac{x}{2}\right)^{\frac{1}{2}-\nu} Q_{\nu}(x)$ bounded on $\mathbf{R}^{+}$provided that $0<\nu \leq \frac{1}{2}$. Using these functions (3.4) takes the form (recall $b=\beta^{2}>0, \nu>0$ )

$$
G_{b, \nu}(r, s)=\frac{\pi}{2}(r s)^{1 / 2}\left[\left(\frac{r}{s}\right)^{\nu} F_{\nu}(\beta r) Q_{\nu}(\beta s)-\left(\frac{s}{r}\right)^{\nu} F_{\nu}(\beta s) Q_{\nu}(\beta r)\right]
$$

Suppose that $0<b \leq b_{0}$ so that $0<\beta \leq b_{0}^{1 / 2}$ and $\tilde{G}_{b, \nu}=b_{0}^{1 / 2} G_{b, \nu}$. If $0<\nu \leq \frac{1}{2}$ then both $F_{\nu}$ and $Q_{\nu}$ are bounded on $\mathbf{R}^{+}$. Hence there is a constant $C=C(\nu)$, which is independent of $b, R, \delta$ and $\varepsilon$, such that

$$
\left|\tilde{G}_{b, \nu}(r, s)\right| \leq C s \quad\left(0<\nu \leq \frac{1}{2}, \quad 0<b \leq b_{0}, \quad 0 \leq r \leq s<+\infty\right)
$$

If however $\nu>\frac{1}{2}$ then $Q_{\nu}$ is not bounded on $\mathrm{R}^{+}$but $Q_{\nu}(\beta x) / x^{\nu-\frac{1}{2}}$ is bounded independent of $\beta \leq b_{0}^{1 / 2}$ for $x \geq R>0$. Hence for any $R>0$ there is a constant $C_{R}=C_{R}(\nu)$, which is independent of $b, \delta$ and $\varepsilon$, and which grows without bound as $R \rightarrow 0^{+}$, such that

$$
\left|\tilde{G}_{b, \nu}(r, s)\right| \leq C_{R} s^{\nu+\frac{1}{2}} \quad\left(\nu>\frac{1}{2}, \quad 0<b \leq b_{0}, \quad 0<R \leq r \leq s<\infty\right)
$$

Suppose that $b>b_{0}$. Then $\tilde{G}_{b, \nu}(r, s)=\beta G_{b, \nu}(r, s)=\frac{\pi}{2}\left[(\beta r)^{\nu+\frac{1}{2}} F_{\nu}(\beta r)(\beta s)^{\frac{1}{2}-\nu} Q_{\nu}(\beta s)\right.$ $\left.-(\beta s)^{\nu+\frac{1}{2}} F_{\nu}(\beta s)(\beta r)^{\frac{1}{2}-\nu} Q_{\nu}(\beta r)\right]$. Since $\left(\frac{x}{2}\right)^{\nu+\frac{1}{2}} F_{\nu}(x)$ and $\left(\frac{x}{2}\right)^{\frac{1}{2}-\nu} Q_{\nu}(x)$ are both bounded on $\mathbf{R}^{+}$when $0<\nu \leq \frac{1}{2}$, there is a constant $C=C(\nu)$, which is independent of $b, R, \delta$ and $\varepsilon$, such that

$$
\left|\tilde{G}_{b, \nu}(r, s)\right| \leq C \quad\left(0<\nu \leq \frac{1}{2}, \quad b>b_{0}, \quad 0 \leq r \leq s<+\infty\right)
$$

When $\nu>\frac{1}{2}$ we only have $\left(\frac{x}{2}\right)^{\frac{1}{2}-\nu} Q_{\nu}(x)$ bounded on $[R,+\infty)$ for $R>0$. Hence for any $R>0$ there is a constant $C_{R}=C_{R}(\nu)$, which is independent of $b, \delta$ and $\varepsilon$, and growing without bound as $R \rightarrow 0^{+}$, such that

$$
\left|\tilde{G}_{b, \nu}(r, s)\right| \leq C_{R} \quad\left(\nu>\frac{1}{2}, \quad b>b_{0}, \quad 0<R \leq r \leq s<+\infty\right)
$$


Next we suppose that $-b_{0} \leq b=-\beta^{2}<0$. In this case $\tilde{G}_{b, \nu}=b_{0}^{1 / 2} G_{b, \nu}$ with $G_{b, \nu}$ given in (3.7). We now also restrict $\delta$ to lie in the range $0<\delta<\beta$. As before we define two related functions $F_{\nu}(x)=e^{-x}\left(\frac{x}{2}\right)^{-\nu} I_{\nu}(x), Q_{\nu}(x)=e^{x}\left(\frac{x}{2}\right)^{\nu} K_{\nu}(x)$ for $\nu>0$. From the known asymptotics we obtain $F_{\nu}(x)=\mathcal{O}(1), Q_{\nu}(x)=\mathcal{O}(1)$ as $x \rightarrow 0^{+}$and $F_{\nu}(x)=\mathcal{O}\left(x^{-\nu-\frac{1}{2}}\right), Q_{\nu}(x)=\mathcal{O}\left(x^{\nu-\frac{1}{2}}\right)$ as $x \rightarrow+\infty$. In terms of these functions (3.7) becomes

$$
G_{b, \nu}(r, s)= \begin{cases}-(r s)^{1 / 2}\left(\frac{s}{r}\right)^{\nu} e^{-\beta(r-s)} F_{\nu}(\beta s) Q_{\nu}(\beta r), & R \leq s \leq r<+\infty, \\ -(r s)^{1 / 2}\left(\frac{r}{s}\right)^{\nu} e^{-\beta(s-r)} F_{\nu}(\beta r) Q_{\nu}(\beta s), & R \leq r \leq s<+\infty .\end{cases}
$$

Notice that as before $F_{\nu} \in L^{\infty}\left(\mathbf{R}^{+}\right)$, and if $0<\nu \leq \frac{1}{2}$ then $Q_{\nu} \in L^{\infty}\left(\mathbf{R}^{+}\right)$. Hence there is a constant $C=C(\nu)$, which is independent of $b, R, \delta$ and $\varepsilon$, such that

$$
\left|\tilde{G}_{b, \nu}(r, s)\right| \leq\left\{\begin{array}{lll}
C r e^{-\beta(r-s)} & \left(0<\nu \leq \frac{1}{2},\right. & \left.-b_{0} \leq b<0, \quad 0 \leq s \leq r<+\infty\right) \\
C s e^{-\beta(s-r)} & \left(0<\nu \leq \frac{1}{2}, \quad-b_{0} \leq b<0, \quad 0 \leq r \leq s<+\infty\right)
\end{array}\right.
$$

If $\nu>\frac{1}{2}$ then $Q_{\nu}$ is no longer bounded on $\mathbf{R}^{+}$, but $Q_{\nu}(\beta x) / x^{\nu-\frac{1}{2}}$ is bounded independent of $\beta \leq b_{0}^{1 / 2}$ for $x \geq R>0$. Thus for any $R>0$ there is a constant $C_{R}=C_{R}(\nu)$, which is independent of $b, \delta$ and $\varepsilon$, and growing without bound as $R \rightarrow 0^{+}$, such that

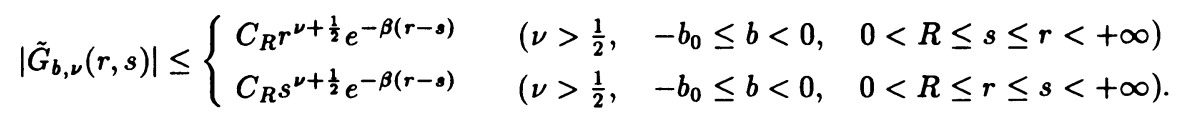

The remaining cases, $b=0$ or $\nu=0$, are in fact limiting cases of those considered above. For brevity we simply state that (3.11) and (3.12) apply when $b=0$, and (3.11), (3.13), and (3.15) apply when $\nu=0$.

LEMMA 3.1. Let $b_{0} \geq 1$ be a given constant, and let $\varepsilon, \delta, R>0$ with $\varepsilon<\delta$. Suppose that $b \geq-b_{0}, \sigma \geq-\frac{1}{2}$, and $p(r)=b-\left(\sigma^{2}-\frac{1}{2}\right) / r^{2}$. If $b<0$ also assume that $\delta<\beta=|b|^{1 / 2}$. Then the solutions of (2.1)-(2.2), given by (3.3) with $G_{b,|\sigma|}$ given by (3.4) when $b>0$ and (3.5) when $b=0$, or given by (3.6)-(3.7) when $b<0$, all satisfy

$$
\max \left(b_{0}^{1 / 2},|b|^{1 / 2}\right)\|w\|_{L_{\delta-c}^{2}(R,+\infty)} \leq C(b, R, \delta, \varepsilon,|\sigma|)\|h\|_{L_{\delta}^{2}(R,+\infty)}
$$

where $C(b, R, \delta, \varepsilon,|\sigma|)$ is a constant of the type described in (3.10).

PROOF: First consider the case $b \geq 0$. According to estimates (3.11)-(3.14) we have $\left|\tilde{G}_{b,|\sigma|}(r, s)\right|^{2} \leq C s^{p}$, for a constant $C$ independent of $b, \delta$, and $\varepsilon$, and power $p$ with $0 \leq p \leq$ $\max (2,2|\sigma|+1)$. Using the inequality $(r+s)^{p} \leq 2^{p}\left(r^{p}+s^{p}\right)$ we have

$$
\int_{r}^{+\infty} s^{p} e^{-2 \delta(s-r)} d s=\int_{0}^{+\infty}(s+r)^{p} e^{-2 \delta s} d s \leq c_{1}+c_{2} r^{p}
$$

for constants $c_{1}, c_{2}>0$ depending on $\delta$ and $p$ but independent of $b, R$ and $\varepsilon$. Since $r^{p} \exp (-2 \varepsilon r)$ is integrable on $\mathbf{R}^{+}$for $p \geq 0, \varepsilon>0$ it follows that the integral in (3.9) is bounded by a constant $C(R, \delta, \varepsilon,|\sigma|)$ independent of $b$. If $|\sigma| \leq \frac{1}{2}$ then the bound on $\tilde{G}_{b,|\sigma|}$ is valid for $0 \leq r \leq s<+\infty$. Therefore we may let $R \rightarrow 0^{+}$in (3.9) without loss of convergence in the integral, and take $C(\varepsilon, \delta,|\sigma|)$ independent of $R$. 
When $b=-\beta^{2}<0$, and $0<\delta<\beta$, it follows from (3.15)-(3.16) that $\left|\tilde{G}_{b,|\sigma|}(r, s)\right|^{2} \leq$ $C r^{p} e^{-2 \beta(r-s)}, s \leq r$, and $\left|\tilde{G}_{b,|\sigma|}(r, s)\right|^{2} \leq C s^{p} e^{-2 \beta(s-r)}, r \leq s$, for a constant $C$ independent of $b, \delta$ and $\varepsilon$, and a power $p$ as above. Hence

$$
\begin{aligned}
& \int_{R}^{+\infty}\left|\tilde{G}_{b,|\sigma|}(r, s)\right|^{2} e^{-2 \delta(s-r)} d s \\
& \quad \leq C \int_{R}^{r} r^{p} e^{-2(\beta-\delta)(r-s)} d s+C \int_{r}^{+\infty} s^{p} e^{-2(\beta+\delta)(s-r)} d s \leq c_{1}+c_{2} r^{p}
\end{aligned}
$$

where $c_{1}, c_{2}>0$ are constants depending on $\delta, p$, and the difference $\beta-\delta$, but independent of $\varepsilon$, $R$. Again it follows that the integral in (3.9) is bounded by a constant

$C=C(\beta-\delta, R, \delta, \varepsilon,|\sigma|)$ which in this case depends also on the difference $\beta-\delta$. If $|\sigma| \leq \frac{1}{2}$ then the integral remains convergent with $R=0$ and $C=C(\beta-\delta, \delta, \varepsilon,|\sigma|)$ can be taken independent of $R$. Estimate (3.17) now follows from (3.8)-(3.9).

\section{A PRIORI ESTIMATES.}

The purpose of this section is to establish a priori estimates on certain solutions of (2.1)-(2.2). Throughout we assume that $p(r)=b-q(r)$ in (2.1) with $b \in \mathbf{R}$ and $q(r)=r^{-2}\left(\sigma^{2}-\frac{1}{4}\right)$ for $\sigma \geq-\frac{1}{2}$. Also we assume $\delta>0$ and if $b<0$ then $\delta<|b|^{1 / 2}$. It is crucial for later developments that the constants involved in these estimates be independent of $b$, for $b \rightarrow+\infty$. It will be convenient for our purposes to assume $b \geq-b_{0}$ for some fixed $b_{0} \geq 1$. We begin with some technical lemmas showing that it is sufficient to establish $L_{\delta}^{2}(R,+\infty)$ estimates on solutions in order to obtain $H_{\delta}^{1}(R,+\infty)$ estimates. The first is a variant of a well-known interpolation estimate.

LEMMA 4.1. Let $\gamma \geq 1$ be given and suppose $\delta \geq 0, R \geq 0$. Then for all $w \in H_{\delta}^{2}(R,+\infty)$ there is a constant $C_{0}$ independent of $\gamma, \delta, R$ such that

$$
\left\|w^{\prime}\right\|_{L_{6}^{2}(R,+\infty)} \leq C_{0}\left[\max \left(1, \delta^{2}\right) \gamma\|w\|_{L_{6}^{2}(R,+\infty)}+\gamma^{-1}\left\|w^{\prime \prime}\right\|_{L_{6}^{2}(R,+\infty)}\right]
$$

PROOF: From [11, p. 70] we know that for any $\varepsilon_{0}>0$ there is a constant $K$, independent of $\varepsilon_{0}$ and $R$, such that for any $f \in H^{2}(R,+\infty)$

$$
\int_{R}^{+\infty}\left|f^{\prime}(r)\right|^{2} d r \leq K \varepsilon_{0}^{-1} \int_{R}^{+\infty}|f(r)|^{2} d r+K \varepsilon_{0} \int_{R}^{+\infty}\left|f^{\prime \prime}(r)\right|^{2} d r .
$$

Taking $f(r)=\exp (\delta r) w(r)$ and $\varepsilon_{0}=\gamma^{-2} \varepsilon$ for some $\varepsilon>0$ yields

$$
\left\|w^{\prime}\right\|^{2}+2 \delta\left(w, w^{\prime}\right)+\delta^{2}\|w\|^{2} \leq K \gamma^{2} \varepsilon^{-1}\|w\|^{2}+4 K \gamma^{-2} \varepsilon\left(\left\|w^{\prime \prime}\right\|^{2}+4 \delta^{2}\left\|w^{\prime}\right\|^{2}+\delta^{4}\|w\|^{2}\right)
$$

where all norms are $L_{\delta}^{2}(R,+\infty)$ norms, and the pairing $\left(w, w^{\prime}\right)$ is the inner product in $L_{\delta}^{2}(R,+\infty)$. For any $\eta>0$ we have $2\left|\left(w, w^{\prime}\right)\right| \leq \eta^{-1}\|w\|^{2}+\eta\left\|w^{\prime}\right\|^{2}$, so that $2 \delta\left(w, w^{\prime}\right) \geq-\delta\left(\eta^{-1}\|w\|^{2}+\eta\left\|w^{\prime}\right\|^{2}\right)$. Since $\gamma^{-1} \leq 1$ it follows that

$$
\left(1-\delta \eta-16 K \delta^{2} \varepsilon\right)\left\|w^{\prime}\right\|^{2} \leq\left(K \gamma^{2} \varepsilon^{-1}+4 K \varepsilon \delta^{4}+\delta \eta^{-1}\right)\|w\|^{2}+4 K \gamma^{-2} \varepsilon\left\|w^{\prime \prime}\right\|^{2} .
$$

We now make the choice $\eta=(3 \delta)^{-1}$ and require that $16 K \delta^{2} \varepsilon \leq \frac{1}{2}$ to obtain

$$
\frac{1}{3}\left\|w^{\prime}\right\|^{2} \leq \gamma^{2}\left(K \varepsilon^{-1}+4 K \varepsilon \delta^{4}+\delta \eta^{-1}\right)\|w\|^{2}+4 K \varepsilon \gamma^{-2}\left\|w^{\prime \prime}\right\|^{2} .
$$

In particular when $\delta \leq 1$ we can set $\varepsilon=(48 K)^{-1}$, while if $\delta>1$ we set $\varepsilon=\left(48 K \delta^{2}\right)^{-1}$. After simplifying and taking square-roots, we finally obtain (4.1). 
LEMMA 4.2. Let $b_{0} \geq 1$ be given. Suppose that $w(r)$ is a solution of (2.1)-(2.2), and that $\max \left(b_{0}^{1 / 2},|b|^{1 / 2}\right)\|w\|_{L_{6}^{2}(R,+\infty)} \leq C\|h\|_{L_{6}^{2}(R,+\infty)}$ for a constant $C=C(b, R, \delta, \varepsilon,|\sigma|)$. Then there is another constant $C=C(b, R, \delta, \varepsilon,|\sigma|)$, having the same type of dependence on the indicated parameters as the first constant, such that

$$
\left\|w^{\prime}\right\|_{L_{6}^{2}(R,+\infty)} \leq C(1+|q(R)|)\|h\|_{L_{6}^{2}(R,+\infty)} .
$$

PROOF: From (2.1), the Minkowski inequality, and the assumed estimate we have

$$
\begin{aligned}
\left\|w^{\prime \prime}\right\|_{L_{6}^{2}(R,+\infty)} & \leq\|h\|_{L_{6}^{2}(R,+\infty)}+\left[\max \left(b_{0},|b|\right)+\frac{\left|\sigma^{2}-\frac{1}{4}\right|}{R^{2}}\right]\|w\|_{L_{6}^{2}(R,+\infty)} \\
& \leq \max \left(b_{0}^{1 / 2},|b|^{1 / 2}\right)\left\{1+C\left(1+\frac{\left|\sigma^{2}-\frac{1}{4}\right|}{R^{2}}\right)\right\}\|h\|_{L_{6}^{2}(R,+\infty)}
\end{aligned}
$$

Hence for a constant $C_{1}=1+C(1+|q(R)|)$ and with $\gamma=\max \left(b_{0}^{1 / 2},|b|^{1 / 2}\right)$ in $(4.1)$ it follows that

$$
\begin{aligned}
\left\|w^{\prime}\right\|_{L_{6}^{2}(R,+\infty)} & \leq C_{0}\left[\max \left(1, \delta^{2}\right) \max \left(b_{0}^{1 / 2},|b|^{1 / 2}\right)\|w\|_{L_{6}^{2}(R,+\infty)}+C_{1}\|h\|_{L_{6}^{2}(R,+\infty)}\right] \\
& \leq C_{0}\left(C \max \left(1, \delta^{2}\right)+C_{1}\right)\|h\|_{L_{\delta}^{2}(R,+\infty)} .
\end{aligned}
$$

This verifies (4.2).

According to Theorem 2.3 and its corollary, problem (2.1)-(2.2) has a unique solution $\widehat{w}(r)$ when $0 \leq \varepsilon<\delta$ and $b \geq 0$. Since $w(r)$, as given by (3.3) and (3.4) or (3.5) respectively, is a solution of (2.1) that has been shown to satisfy (2.2) we have $\widehat{w}(r)=w(r)$. When $b<0$ there is no longer a unique solution of the problem, but we have two particular solutions, $\widehat{w}(r)$ and $w(r)$ as given by (3.6)-(3.7), satisfying estimates (2.9) and (3.17) respectively. Notice that (3.17) holds for $R>0$ while (2.9) holds only for $R_{0}$ sufficiently large. It is our purpose subsequently to show that a unique representative solution can be selected by means of projection operators, and that this solution satisfies both of these estimates. This in turn leads to $H_{\delta}^{1}$ estimates on solutions. We first show that $\widehat{w}(r)$ satisfies the same estimate (3.17) as the function $w(r)$ defined in (3.6)-(3.7).

LEMMA 4.3. Let $b_{0} \geq 1$ be fixed, and let $\varepsilon, \delta, R>0$ with $\varepsilon<\delta$. Suppose that $b \geq-b_{0}$, $\sigma \geq-\frac{1}{2}$, and $p(r)=b-\left(\sigma^{2}-\frac{1}{4}\right) / r^{2}$ in (2.1). If $b<0$ we also assume $\delta+2 \varepsilon<\beta=|b|^{1 / 2}$. Then the function $\widehat{w}$ of Corollary 2.4 satisfies

$$
\max \left(b_{0}^{1 / 2},|b|^{1 / 2}\right)\|\widehat{w}\|_{L_{b-\varepsilon}^{2}(R,+\infty)} \leq C(b, R, \delta, \varepsilon,|\sigma|)\|h\|_{L_{\delta}^{2}(R,+\infty)}
$$

for a constant $C(b, R, \delta, \varepsilon,|\sigma|)$ with the properties described in (3.10).

PROOF: As pointed out above this follows immediately by uniqueness when $b \geq 0$. Suppose $b=-\beta^{2}<0$ and $0<\varepsilon<\delta<\delta+2 \varepsilon<\beta$. Since $b \geq-b_{0}$ it suffices to show (4.3) holds with $\max \left(b_{0}^{1 / 2},|b|^{1 / 2}\right)=1$. Let $w(r)$ be given by (3.6)-(3.7). Then there is a unique $\alpha \in \mathbf{R}$ such that $\widehat{w}(r)=w(r)+\alpha r^{1 / 2} K_{\sigma}(\beta r)$. For convenience we write $\sigma$ rather than $|\sigma|$. Let $R_{0}>0$ be the number guaranteed to exist by Theorem 2.3. Then $\alpha$ must satisfy (note that $w \in L_{\delta}^{2}(R,+\infty)$ )

$$
\alpha=k^{-1} \int_{R_{0}}^{+\infty}[\widehat{w}(r)-w(r)] r^{1 / 2} K_{\sigma}(\beta r) e^{2 \delta r} d r, \quad k=\left\|(\cdot)^{1 / 2} K_{\sigma}(\beta(\cdot))\right\|_{L_{6}^{2}\left(R_{0},+\infty\right)}
$$


By writing $2 \delta=(\delta-\varepsilon)+(\delta+\varepsilon)$ and using the Schwarz inequality we find that

$$
k|\alpha| \leq\|\widehat{w}-w\|_{L_{\sigma-\varepsilon}^{2}\left(R_{0},+\infty\right)}\left\|(\cdot)^{1 / 2} K_{\sigma}(\beta(\cdot))\right\|_{L_{6+\varepsilon}^{2}\left(R_{0},+\infty\right)} .
$$

Since $\beta>\delta+2 \varepsilon$ and $K_{\sigma}(\beta r)$ is monotonically decreasing we have $K_{\sigma}(\beta r) \leq K_{\sigma}(\gamma r)$, where $\gamma=\delta+2 \varepsilon$ for convenience. Using $Q_{\sigma}(x)=e^{x}\left(\frac{x}{2}\right)^{\sigma} K_{\sigma}(x), \sigma>0$, as defined previously, and noting that $K_{\sigma}(x)$ is a positive function it follows that

$$
\left\|(\cdot)^{1 / 2} K_{\sigma}(\beta(\cdot))\right\|_{L_{\sigma+\varepsilon}^{2}\left(R_{0},+\infty\right)}^{2} \leq \int_{R_{0}}^{+\infty} r\left(\frac{\gamma r}{2}\right)^{-2 \sigma}\left|Q_{\sigma}(\gamma r)\right|^{2} e^{-2(\gamma-\delta-\varepsilon) r} d r .
$$

Clearly $\gamma r \geq(\delta+2 \varepsilon) R_{0}>0$ so that $(\gamma r)^{-2 \sigma}$ and $\left|Q_{\sigma}(\gamma r)\right|$ are both bounded at the lower limit. Also $\gamma-\delta-\varepsilon=\varepsilon>0$ and $Q_{\sigma}$ has at most polynomial growth at infinity; thus the integral on the right is convergent. Let $C\left(R_{0}, \delta, \varepsilon, \sigma\right)$ denote its value. Then from (2.9) and (3.17) it follows that

$$
\begin{aligned}
k|\alpha| & \leq C\left(\sigma, \delta, \varepsilon, R_{0}\right)\|\hat{w}-w\|_{L_{\delta-\varepsilon}^{2}\left(R_{0},+\infty\right)} \\
& \leq C\left(\sigma, \delta, \varepsilon, R_{0}\right)\left(\|\widehat{w}\|_{L_{6}^{2}\left(R_{0},+\infty\right)}+\|w\|_{L_{6-\varepsilon}^{2}(R,+\infty)}\right) \leq C(b, R, \delta, \varepsilon, \sigma)\|h\|_{L_{\delta}^{2}(R,+\infty)}
\end{aligned}
$$

for a constant $C(b, R, \delta, \varepsilon, \sigma)$ as described in (3.10). Now changing variables $x=\beta r$ in the integral defining $k$ and using $\beta \leq b_{0}^{1 / 2}$ shows immediately that $k$ is bounded away from zero independent of $\beta=|b|^{1 / 2}$. Thus $|\alpha| \leq C\|h\|_{L_{6}^{2}(R,+\infty)}$ for a constant $C$ as above. It now follows from this, (3.17), and the triangle inequality that $\widehat{w}$ satisfies (4.3).

We now introduce the projection operators $Q_{\beta}^{m}$, which will be used in determining unique solutions. Let $0<\delta<\beta$ and suppose that $\sigma \geq 0$. Let $H_{\delta}^{m}(R,+\infty)=\left\{w \in L_{\delta}^{2}(R,+\infty)\right.$ : $\left.w^{(k)} \in L_{\delta}^{2}(R,+\infty), 0 \leq k \leq m\right\}$ be the weighted Sobolev space as defined in Section 1. Since $\beta>\delta$ we have $r^{1 / 2} K_{\delta}(\beta r) \in H_{\delta}^{m}(R,+\infty), R>0$. We define $Q_{\beta}^{m}$ to be the orthogonal projection in $H_{\delta}^{m}(R,+\infty)$ onto the 1-dimensional subspace spanned by $r^{1 / 2} K_{\sigma}(\beta r)$. Of course $Q_{\beta}^{m}$ also depends on $R, \delta$ and $\sigma$. If $0 \leq \sigma \leq \frac{1}{2}$ and $m=0$ then $Q_{\beta}^{m}$ is also well-defined for $R=0$. In general, however, we must have $R>0$. Computationally, we have $Q_{\beta}^{m} w(r)=\alpha r^{1 / 2} K_{\sigma}(\beta r)$ where

$$
\alpha=\left\|(\cdot)^{1 / 2} K_{\sigma}(\beta(\cdot))\right\|_{H_{6}^{m}(R,+\infty)}^{-2}\left(w,(\cdot)^{1 / 2} K_{\sigma}(\beta(\cdot))\right)_{H_{\sigma}^{m}(R,+\infty)} .
$$

THEOREM 4.4. Let $b_{0} \geq 1$ be a given constant, and in (2.1) let $p(r)=b-q(r)$ where $b \geq-b_{0}$ and $q(r)=\left(\sigma^{2}-\frac{1}{4}\right) / r^{2}$ with $\sigma \geq-\frac{1}{2}$. If $|\sigma| \leq \frac{1}{2}$ let $R=0$, otherwise assume $R>0$. Suppose that $\varepsilon=\delta$ in (2.2).

i) If $b \geq 0$ and $0<\delta<+\infty$ then for every $h \in L_{\delta}^{2}(R,+\infty)$ there is a unique solution $w^{*}$ of (2.1)-(2.2).

ii) If $b<0$ and $0<\delta<\beta=|b|^{1 / 2}$ then for every $h \in L_{\delta}^{2}(R,+\infty)$ problem (2.1)-(2.2) has a 1-dimensional affine space of solutions. All solutions have the form $w(r)=\alpha r^{1 / 2} K_{|\sigma|}(\beta r)+$ $w^{*}(r)$, where $\alpha \in \mathbf{R}$ and $w^{*}$ is the unique solution satisfying $Q_{\beta}^{0} w=0$. 
Moreover in either case there is a constant $C=C(b, R, \delta,|\sigma|)$, having the properties described in (3.10), but being independent of $\varepsilon$, such that the following estimates hold:

$$
\begin{gathered}
\max \left(b_{0}^{1 / 2},|b|^{1 / 2}\right)\left\|w^{*}\right\|_{L_{6}^{2}(R,+\infty)} \leq C\|h\|_{L_{6}^{2}(R,+\infty)}, \\
\left\|w^{*}\right\|_{H_{b}^{1}(R,+\infty)} \leq C(1+|q(R)|)\|h\|_{L_{6}^{2}(R,+\infty)}, \quad R>0, \\
\left|w^{*}(r)\right| \leq C e^{-\delta r}(1+|q(R)|)\|h\|_{L_{6}^{2}(R,+\infty)}, \quad r>R>0, \\
\left|w^{* \prime}(r)\right|,|b|^{1 / 2}\left|w^{*}(r)\right| \leq C e^{-\delta r}(1+|q(R)|)\|h\|_{L_{6}^{2}(R,+\infty)}, \quad r>R>0 .
\end{gathered}
$$

PROOF: Suppose that $b \geq 0$. Let $\widehat{w}$ denote the unique solution of Corollary 2.4i, and let $w$ be the solution given by (3.3) and (3.4) or (3.5) respectively. Since $w$ satisfies (2.1)-(2.2) with $0<\varepsilon<\delta$ we have $\widehat{w}=w$. Suppose that $u(r)$ is a solution of (2.1)-(2.2) with $\varepsilon=\delta$. Then $v(r)=\widehat{w}(r)-u(r)$ is a solution of the corresponding homogeneous equation which satisfies (2.2) with $\varepsilon=\delta$. From the form that $v$ must take (cf. Section 3 ) it follows that $v$ is identically zero. Therefore (2.1)-(2.2), with $\varepsilon=\delta$, has a unique solution $w^{*}=\widehat{w}=w$ where $w$ is given by (3.3) and (3.4) or (3.5) respectively.

To verify (4.5) we let $R_{0}>0$ be the number guaranteed to exist by Theorem 2.3 , and choose $\varepsilon=\delta / 2$. Clearly we have

$$
\begin{aligned}
\left\|w^{*}\right\|_{L_{\delta}^{2}(R,+\infty)}^{2} & \leq e^{2 \varepsilon R_{0}} \int_{R}^{R_{0}}\left|w^{*}(r)\right|^{2} e^{2(\delta-\varepsilon) r} d r+\int_{R_{0}}^{+\infty}\left|w^{*}(r)\right| e^{2 \delta r} d r \\
& \leq e^{2 \varepsilon R_{0}}\left\|w^{*}\right\|_{L_{\delta-\varepsilon}^{2}(R,+\infty)}^{2}+\left\|w^{*}\right\|_{L_{\delta}^{2}\left(R_{0},+\infty\right)}^{2}
\end{aligned}
$$

Thus from (2.9) and (4.3) we obtain

$$
\max \left(b_{0}^{1 / 2}, b^{1 / 2}\right)\left\|w^{*}\right\|_{L_{6}^{2}(R,+\infty)} \leq C\|h\|_{L_{6}^{2}(R,+\infty)}
$$

for a constant $C=C(R, \delta,|\sigma|)$. If $|\sigma| \leq \frac{1}{2}$ then we can allow $R=0$ and take $C$ independent of $R$. We now apply Lemma 4.2 to obtain (4.6).

If $b=-\beta^{2}<0$ and $0<\varepsilon<\delta$ then all solution of (2.1)-(2.2) have the form $w(r)=\widehat{w}(r)+$ $\alpha r^{1 / 2} K_{\sigma}(\beta r)$ as described in Theorem 2.3. Again we write $\sigma$ rather than $|\sigma|$. Since $\delta<\beta$ all of these solutions belong to $L_{\delta}^{2}(R,+\infty)$ with $R>0$ if $\sigma>\frac{1}{2}$, and with $R=0$ if $0 \leq \sigma \leq \frac{1}{2}$. If $u(r)$ is a solution of (2.1)-(2.2) with $\varepsilon=\delta$ then $v(r)=\widehat{w}(r)-u(r)$ satisfies (2.1) with $h$ identically zero. Thus $v(r)=c_{1} r^{1 / 2} K_{\sigma}(\beta r)+c_{2} r^{1 / 2} I_{\sigma}(\beta r)$ for some $c_{1}, c_{2} \in \mathbf{R}$. But then $v(r)-c_{1} r^{1 / 2} K_{\sigma}(\beta r)$ belongs to $L^{2}(R,+\infty)$ which implies $c_{2}=0$. Thus $u(r)=\widehat{w}(r)-c_{1} r^{1 / 2} K_{\sigma}(\beta r)$. This shows all solutions of (2.1)-(2.2), with $\varepsilon=\delta$, have the form $w(r)=\widehat{w}(r)+\alpha r^{1 / 2} K_{\sigma}(\beta r)$.

Now set $w^{*}=\left(I-Q_{\beta}^{0}\right) \hat{w}$. Clearly $w^{*}$ is well-defined and satisfies (2.1)-(2.2) when $\varepsilon=\delta$. Moreover $\left\|w^{*}\right\| \leq\|\widehat{w}\|$ in the $L_{\delta}^{2}(R,+\infty)$ norm since $Q_{\beta}^{0}$ is an orthogonal projection. By choosing $\varepsilon=\min \left(\frac{\delta}{2}, \frac{(\beta-\delta)}{4}\right)$ we have $0<\varepsilon<\delta<\delta+2 \varepsilon<\beta$. Hence we can repeat the argument above leading to (4.9), with $\widehat{w}$ replacing $w^{*}$. Then using $\left\|w^{*}\right\| \leq\|\widehat{w}\|$ yields (4.9). The estimate (4.6) then follows from Lemma 4.2 .

To obtain (4.7) we use (4.5) and the fact that $w^{*}(r) \rightarrow 0$ as $r \rightarrow+\infty$ to estimate

$$
\left|w^{*}(r)\right| \leq \int_{r}^{+\infty}\left|w^{* \prime}(s)\right| d s \leq \delta^{-1} e^{-\delta r}\left\|w^{* \prime}\right\|_{L_{6}^{2}(R,+\infty)} \leq C e^{-\delta r}(1+|q(R)|)\|h\|_{L_{6}^{2}(R,+\infty)} .
$$

To obtain the two remaining estimates we begin by multiplying $(2.1)$ by $w^{\prime}$ where for convenience we write $w$ in place of $w^{*}$. Integrating from $r$ to $+\infty$, and using the limits $w(r), w^{\prime}(r) \rightarrow 0$ as 
$r \rightarrow+\infty$, we obtain (with $\left.q(s)=\left(\sigma^{2}-\frac{1}{4}\right) / s^{2}\right)$

$$
\left|w^{\prime}(r)\right|^{2}+b|w(r)|^{2}=-2\left[\int_{r}^{+\infty} h(s) w^{\prime}(s) d s+\int_{r}^{+\infty} q(s) w(s) w^{\prime}(s) d s\right]
$$

By the previous estimate it suffices to consider $b \geq b_{0}$. Bounding the first integral on the right we have

$$
\begin{aligned}
\left|2 \int_{r}^{+\infty} h(s) w^{\prime}(s) d s\right| & \leq 2 \int_{r}^{+\infty}|h(s)| e^{\delta s}\left|w^{\prime}(s)\right| e^{\delta s} e^{-2 \delta s} d s \\
& \leq e^{-2 \delta r}\left(\|h\|_{L_{6}^{2}(R,+\infty)}^{2}+\left\|w^{\prime}\right\|_{L_{6}^{2}(R,+\infty)}^{2}\right) \\
& \leq C e^{-2 \delta r}(1+|q(R)|)\|h\|_{L_{6}^{2}(R,+\infty)}^{2}
\end{aligned}
$$

for a constant $C=C(b, R, \delta, \sigma)$ as described above. Similarly for the second integral we find

$$
\left|2 \int_{r}^{+\infty} q(s) w(s) w^{\prime}(s) d s\right| \leq C|q(r)| e^{-2 \delta r}(1+|q(R)|)\|h\|_{L_{6}^{2}(R,+\infty)}^{2} .
$$

Estimate (4.8) now follows.

In the preceeding theorem it was shown that there is a uniquely determined function $w^{*}$ satisfying (2.1)-(2.2). When $b<0$ the uniqueness property was obtained by adding the constraint

$$
Q_{\beta}^{m} w=0
$$

with $m=0$. Since $Q_{\beta}^{m}$ is well-defined for all $m \geq 0$ when $R>0$ it is clear that uniqueness can be obtained by adding the constraint (4.10) for any choice of $m \leq 2$. By the nature of the a priori estimates (4.5)-(4.8) we have established it turns out that the case $m=1$ also allows a passage to the limit to the original problem (1.4)-(1.6). Thus we also record the following.

THEOREM 4.5. Under the hypotheses of Theorem 4.4, with the exception that $R>0$ is now required unless $|\sigma|=1 / 2$, all conclusions of Theorem 4.4 remain valid with $Q_{\beta}^{1}$ replacing $Q_{\beta}^{0}$.

PROOF: When $b \geq 0$ the results are identical. When $b<0$ we set $\beta=|b|^{1 / 2}$ and $w^{* *}=Q_{\beta}^{1} w^{*}$, where $w^{*}$ is the unique solution obtained via the projection $Q_{\beta}^{0}$. Since $Q_{\beta}^{1}$ is an orthogonal projection in $H_{\delta}^{1}(R,+\infty)$ the inequality (4.6) follows immediately. Since $|b|^{1 / 2} \leq b_{0}^{1 / 2}$ the first inequality (4.5) then follows from (4.6). Having verified (4.5)-(4.6) for $w^{* *}$ we may repeat the same argument as before to obtain (4.7)-(4.8).

\section{Passage to the limit.}

Our goal in this section is to prove existence of solutions and to characterize the set of solutions of problem (1.4)-(1.6). As in Section 1 we set $q_{N}(r)=(N-1)(N-3) / 4 r^{2}$. We begin by considering the finite dimensional approximating problem

$$
\begin{gathered}
\frac{\partial^{2}}{\partial t^{2}} W_{m}-\frac{\partial^{2}}{\partial r^{2}} W_{m}+\left(\lambda+q_{N}(r)\right) W_{m}=H_{m} \\
(t, r) \in(0, T) \times(R,+\infty)
\end{gathered}
$$




$$
\begin{aligned}
& W_{m}(t, \cdot) \in L^{2}(R,+\infty), \quad t \in(0, T) \\
& W_{m}(t+T, r)=W_{m}(t, r), \quad(t, r) \in \mathbf{R} \times(R,+\infty), \quad \text { or } \\
& \begin{cases}\alpha_{1} W_{m}(0, r)-\alpha_{2} \frac{\partial}{\partial t} W_{m}(0, r)=0, & r \in(R,+\infty), \\
\beta_{1} W_{m}(T, r)+\beta_{2} \frac{\partial}{\partial t} W_{m}(T, r)=0, & r \in(R,+\infty),\end{cases}
\end{aligned}
$$

where $R>0$ and $H_{m}(t, r)=\sum_{n \leq m} h_{n}(r) \xi_{n}(t)$. The functions $h_{n}, \xi_{n}$ were defined in Section 1 . We seek a solution $W_{m}(t, r)=\sum^{n} w_{n}(r) \xi_{n}(t)$ having the same form. Let $\sigma=\sigma_{N}=(N-2) / 2$ so that $(N-1)(N-3) / 4 r^{2}=\left(\sigma^{2}-\frac{1}{4}\right) / r^{2}=q_{N}(r)$ as in the previous sections. Recalling that $\xi_{n}^{\prime \prime}+\theta_{n}^{2} \xi_{n}=0$ and that $\xi_{n}$ satisfies the boundary conditions (1.9) we are lead to the componentwise equations

$$
\begin{aligned}
& w_{n}^{\prime \prime}+\left(b_{n}-q_{N}(r)\right) w_{n}=-h_{n}, \quad r>R, \\
& w_{n} \in L^{2}(R,+\infty),
\end{aligned}
$$

in which $b_{n}=\theta_{n}^{2}-\lambda$. There are actually two cases to consider: i) $\lambda \leq \min \theta_{n}^{2}$ and ii) $\theta_{j}^{2}<\lambda \leq \theta_{j+1}^{2}$ for some $j$. However the arguments are similar so for brevity we only consider the more delicate second case. Also, since we are interested in the limit of $W_{m}$ as $m \rightarrow+\infty$ we assume $m>j$. Then for some $b_{0} \geq 1$ we have $-b_{0} \leq b_{n} \leq b_{j}<0$ for $n \leq j$, and $b_{n} \geq 0$ for $n>j$. Set $\beta_{n}=\left|b_{n}\right|^{1 / 2}$ for $n \leq j$. According to Theorem 4.4 all solutions of (5.4)-(5.5) are either uniquely determined when $n>j$, or given by

$$
w_{n}(r)=w_{n}^{*}(r)+\alpha_{n} r^{1 / 2} K_{\sigma}\left(\beta_{n} r\right), \quad \alpha_{n} \in \mathbf{R},
$$

when $n \leq j$. Hence all solutions of (5.1)-(5.3) have the form

$$
W_{m}(t, r)=\sum_{n \leq m} w_{n}^{*}(r) \xi_{n}(t)+\sum_{n \leq j} \alpha_{n} r^{1 / 2} K_{\sigma}\left(\beta_{n} r\right) \xi_{n}(t)
$$

Let $Q_{\beta_{n}}^{0}: L_{\delta}^{2}(R,+\infty) \rightarrow \operatorname{span}\left\{r^{1 / 2} K_{\sigma}\left(\beta_{n} r\right)\right\}$ be the orthogonal projection defined in (4.4), with $m=0$ and $\beta=\beta_{n}$. Then $Q_{\beta_{n}}^{0} w_{n}^{*}=0$ where $w_{n}^{*}$ is the function appearing in (5.6) and defined in Theorem 4.4 .

Clearly the functions

$$
v_{n}(t, r)=r^{1 / 2} K_{\sigma}\left(\beta_{n} r\right) \xi_{n}(t) \quad(n \leq j)
$$

satisfy (5.1)-(5.3) with $H_{m}$ identically zero, and also (1.4)-(1.6) with $h$ identically zero. Let $L_{\lambda}$ denote the (closed unbounded) linear operator associated with problem (1.4)-(1.6), so that $L_{\lambda} w=w_{t t}-w_{r r}+\left(\lambda+q_{N}(r)\right) w$ for smooth functions $w$ satisfying (1.5)-(1.6). Then $v_{n} \in \operatorname{ker}\left(L_{\lambda}\right)$ for $n \leq j$. We define an orthogonal projection $P_{\lambda}: L_{\delta}^{2}((0, T) \times(R,+\infty)) \rightarrow \operatorname{span}\left\{v_{n}\right\}_{n \leq j}$ by (recall $\left.c_{n}=\left\|\xi_{n}\right\|_{L^{2}(0, T)}^{2}\right)$

$$
\left\{\begin{array}{l}
P_{\lambda} w=\sum_{n \leq j} \alpha_{n} r^{1 / 2} K_{\sigma}\left(\beta_{n} r\right) \xi_{n}(t), \quad \text { where } \\
\alpha_{n}=Q_{\beta_{n}}^{0} w_{n}(r) / r^{1 / 2} K_{\sigma}\left(\beta_{n} r\right), \quad w_{n}(r)=c_{n}^{-1} \int_{0}^{T} w(t, r) \xi_{n}(t) d t .
\end{array}\right.
$$

Then $W_{m}^{*}(t, r)=\left(I-P_{\lambda}\right) W_{m}(t, r)$ is the first summand appearing on the right in (5.7), and is a uniquely determined solution of (5.1)-(5.3). Moreover since the functions $\xi_{n}(t)$ are othogonal in $L^{2}(0, T)$ we have

$$
\int_{0}^{T} \int_{R}^{+\infty} w_{k}^{*}(r) \xi_{k}(t) w_{n}^{*}(r) \xi_{n}(t) e^{2 \delta r} d r d t= \begin{cases}c_{k}\left\|w_{k}^{*}\right\|_{L_{\delta}^{2}(R,+\infty)}^{2} & (k=n) \\ 0 & (k \neq n)\end{cases}
$$


Hence from (4.5) it follows that

$$
\begin{aligned}
\left\|W_{m}^{*}\right\|_{L_{\delta}^{2}((0, T) \times(R,+\infty))}^{2} & =\sum_{n \leq m} c_{n}\left\|w_{n}^{*}\right\|_{L_{\delta}^{2}(R,+\infty)}^{2} \\
& \leq c(\delta, \beta,-\delta, N, R) \sum_{n \leq m}\left\|h_{n}\right\|_{L_{6}^{2}(R,+\infty)}^{2} \\
& \leq c(\delta, \beta,-\delta, N, R)\|h\|_{L_{\delta}^{2}((0, T) \times(R,+\infty))}^{2} .
\end{aligned}
$$

Here $c\left(\delta, \beta_{j}-\delta, N, R\right)$ is used to denote a constant depending on the indicated quantities, but independent of $m$ and $h$. In general $c \rightarrow+\infty$ as $\delta \rightarrow 0^{+}$and as $\delta \rightarrow \beta_{j}^{-}$, and also $c \rightarrow+\infty$ as $R \rightarrow 0^{+}$if $N>3$.

From basic Hilbert space theory (cf. [18]) it follows that there is a unique function $w^{*}(t, r)=$ $\lim _{m \rightarrow \infty} W_{m}(t, r)$, belonging to $L_{\delta}^{2}((0, T) \times(R,+\infty))$, with the limit meant in the sense of $L_{\delta}^{2}((0, T) \times(R,+\infty))$ convergence. Also we must have

$$
\left\|w^{*}\right\|_{L_{\delta}^{2}((0, T) \times(R,+\infty))} \leq c(\delta, \beta,-\delta, N, R)\|h\|_{L_{6}^{2}((0, T) \times(R,+\infty))}
$$

and according to (4.5) if $N \leq 3$ we can take $R=0$ and $c=c\left(\delta, \beta_{j}-\delta, N\right)$.

Let $\mathcal{D}_{T, R}$ be the set of test functions defined in section one. Then

$$
\int_{0}^{T} \int_{R}^{+\infty} W_{m}^{*}\left[\varphi_{t t}-\varphi_{r r}+\left(\lambda+q_{N}(r)\right) \varphi\right] d r d t=\int_{0}^{T} \int_{R}^{+\infty} H_{m} \varphi d r d t, \quad \forall \varphi \in \mathcal{D}_{T, R}
$$

Since $\varphi \in \mathcal{D}_{T, R}$ implies $\varphi_{t t}-\varphi_{r r}+\left(\lambda+q_{N}(r)\right) \varphi \in L^{2}((0, T) \times(R,+\infty))$ we may pass to the limit in (5.11) to obtain

$$
\int_{0}^{T} \int_{R}^{+\infty} w^{*}\left[\varphi_{t t}-\varphi_{r r}+\left(\lambda+q_{N}(r)\right) \varphi\right] d r d t=\int_{0}^{T} \int_{R}^{+\infty} h \varphi d r d t, \quad \forall \varphi \in \mathcal{D}_{T, R} .
$$

Hence $w^{*}$ is a solution of (1.4)-(1.6), and depends continuously on $h$ according to (5.10). If $w \in L^{2}((0, T) \times(R,+\infty))$ is another solution then the argument leading to (1.12)-(1.13) shows $w_{n}(r)$, as defined by (1.11), must satisfy (5.4)-(5.5). Hence $w_{n}(r)-w_{n}^{*}(r)=\alpha_{n} r^{1 / 2} K_{\sigma}\left(\beta_{n} r\right)$ for $n \leq j$ and some $\alpha_{n} \in \mathbf{R}$. If $n>j$ then $w_{n}(r)-w_{n}^{*}(r)=0$. Therefore $w=w^{*}+v$ where $v \in \operatorname{span}\left\{v_{n}\right\}$ with $v_{n}$ as defined in (5.8). In particular this shows $\operatorname{ker}\left(L_{\lambda}\right)=\operatorname{span}\left\{v_{n}\right\}_{n \leq J}$. We have verified the following result.

THEOREM 5.1. Suppose that $\theta_{j}^{2}<\lambda \leq \theta_{j+1}^{2}$ for some $j$ and define $\beta_{n}=\left|\theta_{n}^{2}-\lambda\right|^{1 / 2}$, $n \leq j$. Then for all $\delta \in\left(0, \beta_{j}\right)$ and any $h \in L_{\delta}^{2}((0, T) \times(R,+\infty))$ there is a finite dimensional affine space of solutions of (1.4)-(1.6). All solutions have the form $w=w^{*}+v$, where $w^{*}$ is the solution uniquely determined by $P_{\lambda} w^{*}=0$ and $v \in \operatorname{ker}\left(L_{\lambda}\right)$. Furthermore the solution $w^{*}$ satisfies (5.10), and if $N \leq 3$ then $R=0$ is allowed.

For certain choices of the boundary conditions (1.6) it is possible to show that solutions have more regularity than stated above. For instance when the boundary conditions are periodic, Dirichlet, or Neumann we have

$$
\int_{0}^{T} \xi_{n}^{\prime}(t) \xi_{k}^{\prime}(t) d t= \begin{cases}\theta_{n}^{2} c_{n}, & n=k \\ 0, & n \neq k .\end{cases}
$$

Thus for a function of the form $W_{m}(t, r)=\sum_{n \leq m} w_{n}(r) \xi_{n}(t)$ it follow that 


$$
\left\|\frac{\partial}{\partial t} W_{m}\right\|_{L_{\delta}^{2}((0, T) \times(R,+\infty))}^{2}=\sum_{n \leq m} c_{n} \theta_{n}^{2}\left\|w_{n}\right\|_{L_{\delta}^{2}(R,+\infty)}^{2} .
$$

With these choices of the boundary conditions (1.6) in mind we again consider the sequence of problems (5.4)-(5.5). As before we assume $\theta_{j}^{2}<\lambda \leq \theta_{j+1}^{2}$. By applying Theorem 4.5 we find that all solutions have the form (5.7), with $w_{n}^{*}$ uniquely determined by the constraint $Q_{\beta_{n}}^{1} w_{n}^{*}=0$ when $n \leq j$. Here $Q_{\beta_{n}}^{1}$ denotes the projection defined in (4.4) with $m=1$ and $\beta=\beta_{n}$. We now define $P_{\lambda}^{1}: H_{\delta}^{1}((0, T) \times(R,+\infty)) \rightarrow \operatorname{span}\left\{v_{n}\right\}_{n \leq \jmath}$ as in (5.9), but with $Q_{\beta_{n}}^{1}$ replacing $Q_{\beta_{n}}^{0}$. Then as before $W_{m}^{*}=\left(I-P_{\lambda}^{1}\right) W_{m}$ is the first summand on the right in (5.7).

THEOREM 5.2. Suppose that the boundary conditions (1.6) are such that the associated eigenvalue problem (1.8)-(1.9) has eigenfunctions which satisfy (5.13). Assume that $\theta_{j}^{2}<\lambda \leq$ $\theta_{j+1}^{2}$ for some $j$ and define $\beta_{n}=\left|\theta_{n}^{2}-\lambda\right|^{1 / 2}, n \leq j$. Then for all $\delta \in(0, \beta$,$) and any h \in$ $L_{\delta}^{2}((0, T) \times(R,+\infty))$ there is a finite-dimensional affine space of solutions of (1.4)-(1.6). All solutions belong to $H_{\delta}^{1}((0, T) \times(R,+\infty))$ and have the form $w=w^{*}+v$, where $w^{*}$ is the unique solution satisfying $P_{\lambda}^{1} w^{*}=0$ and $v \in \operatorname{ker}\left(L_{\lambda}\right)$. Furthermore $w^{*}$ satisfies the a priori estimates

$$
\begin{aligned}
& \left\|w^{*}\right\|_{H_{\delta}^{1}((0, T) \times(R,+\infty))} \leq c\left(1+\left|q_{N}(R)\right|\right)\|h\|_{L_{\delta}^{2}((0, T) \times(R,+\infty))}, \\
& \left\|w^{*}\right\|_{L_{\delta}^{\infty}((0, T) \times(R,+\infty))} \leq c\left(1+\left|q_{N}(R)\right|\right)\|h\|_{L_{\delta}^{2}((0, T) \times(R,+\infty))},
\end{aligned}
$$

where $c=c(\delta, \beta,-\delta, N, R)$ is a constant with the same properties as in (5.10).

PROOF: The existence and uniqueness of $w^{*}$ follows in the same way as before. To verify (5.15) it remains to show that $w_{t}^{*}, w_{r}^{*}$ exist and satisfy appropriate estimates. First we consider $w_{i}^{*}$. Since $\theta_{n}^{2}=b_{n}+\lambda$ it follows from (5.14), applied to $W_{m}^{*}=\left(I-P_{\lambda}^{1}\right) W_{m}$, and (4.5) that

$$
\left\|\frac{\partial}{\partial t} W_{m}^{*}\right\|_{L_{\delta}^{2}((0, T) \times(R,+\infty))} \leq c\|h\|_{L_{\delta}^{2}((0, T) \times(R,+\infty))},
$$

where $c=c(\delta, \beta,-\lambda, N, R, \lambda)$ now also depends on $\lambda$, but is independent of $m$. This shows that $\frac{\partial}{\partial t} W_{m}$ converges in $L_{\delta}^{2}((0, T) \times(R,+\infty))$, and a standard argument verifies that $w_{t}^{*}=\lim \frac{\partial}{\partial t} W_{m}^{*}$ in the sense of distributions. Essentially the same argument applies to $w_{r}^{*}=\lim \frac{\partial}{\partial r} W_{m}^{*}$, with (4.6) used in place of (4.5).

To verify (5.16) we again use orthogonality, and estimate (4.7), to obtain

$$
\begin{aligned}
\int_{0}^{T}\left|W_{m}^{*}(t, r)\right|^{2} d t & =\sum_{n \leq m} c_{n}\left|w_{n}^{*}(r)\right|^{2} \\
& \leq c e^{-2 \delta r}\left(1+\left|q_{N}(R)\right|\right)^{2} \sum_{n \leq m}\left\|h_{n}\right\|_{L_{\delta}^{2}(R,+\infty)}^{2}
\end{aligned}
$$

Similar estimates apply for $\frac{\partial}{\partial t} W_{m}^{*}$ and $\frac{\partial}{\partial r} W_{m}^{*}$. These combine to give

$$
\begin{gathered}
\left\{\int_{0}^{T}\left(\left|W_{m}^{*}(t, r)\right|^{2}+\left|\frac{\partial}{\partial t} W_{m}^{*}(t, r)\right|^{2}+\left|\frac{\partial}{\partial r} W_{m}^{*}(t, r)\right|^{2}\right) d t\right\}^{1 / 2} \\
\leq c\left(1+\left|q_{N}(R)\right|\right) e^{-\delta r}\|h\|_{L_{6}^{2}((0, T) \times(R,+\infty))}
\end{gathered}
$$

where once again $c=c(\delta, \beta,-\delta, N, R, \lambda)$ is independent of $m$. Now from this estimate and the Sobolev embedding $H^{1}(0, T) \rightarrow C(0, T)$ it follows that (a.e) $(t, r) \in(0, T) \times(R,+\infty)$

$$
\left|W_{m}^{*}(t, r)\right| \leq c\left\|W_{m}^{*}(\cdot, r)\right\|_{H^{1}(0, T)} \leq c e^{-\delta r}\left(1+\left|q_{N}(R)\right|\right)\|h\|_{L_{\delta}^{2}((0, t) \times(R,+\infty))} .
$$

Since $W_{m}^{*}(t, r) \rightarrow w(t, r)($ a.e) $(t, r) \in(0, T) \times(R,+\infty)$, the same estimate holds for $w(t, r)$, which in turn implies (5.16). 
REMARK: When $\lambda \leq \min \theta_{n}$ analogous arguments apply and show there is a unique solution $w^{*} \in H_{\delta}^{1}((0, T) \times(R,+\infty))$ of (1.4)-(1.6) which also satisfies (5.15)-(5.16).

COROLLARY 5.3. The (closed unbounded) linear operator $L_{\lambda} w=w_{t t}-w_{r r}+\left(\lambda+q_{N}(r)\right) w$, where $q_{N}(r)=(N-1)(N-3) / 4 r^{2}$, associated with (1.4)-(1.6) is a Fredholm operator from $\operatorname{dom}\left(L_{\lambda}\right) \subset L^{2}((0, T) \times(R,+\infty))$ onto $L_{\delta}^{2}((0, T) \times(R,+\infty))$ with non-negative index. The parameter $\delta>0$ and the index of $L_{\lambda}$ depend on $\lambda$ in the following way. If $\lambda \leq \min \theta_{n}^{2}$ then $\delta>0$ is otherwise unrestricted and the index is zero. If $\theta_{j}^{2}<\lambda \leq \theta_{j+1}^{2}$ then $0<\delta<\beta,=\left|\lambda-\theta_{j}\right|^{1 / 2}$ and the index is positive and equal to the dimension of the linear space spanned by $\left\{\xi_{n}(t)\right\}_{n \leq J}$. In addition $L_{\lambda}$ has a bounded (partial) inverse $K_{\lambda}: L_{\delta}^{2}((0, T) \times(R,+\infty)) \rightarrow H_{\delta}^{1}((0, T) \times(R,+\infty)) \cap$ $L_{\delta}^{\infty}((0, T) \times(R,+\infty))$ defined by the correspondence $h \rightarrow w^{*}$, where $w^{*}$ is described in Theorem 5.2 .

\section{REFERENCES}

1. SMILEY, M. W. Complex-valued time-periodic solutions and breather of nonlinear wave equations, to appear in Differential and Integral Equations.

2. CORON, J. M. Période minimale pour une corde vibrante de longueur infinie, $\underline{C}$. $R$. Acad. Sci. Paris Ser A 294 (1982), 127-129.

3. LEVINE, H. A. Minimal periods for solutions of semilinear wave quations in exterior domains and for solutions of the equations of nonlinear elasticity, to appear in J. of Math. Anal. \& Appl.

4. STRAUSS, W. A. Stable and unstable states of nonlinear wave equations, in Nonlinear Partial Differential Equations, Contemporary Mathematic Vol. 17, Amer. Math. Soc., Providence, R.I., 1983.

5. VUILLERMOT, $P$. Nonexistence of spatially localized free vibrations for certain semilinear wave equations on $\mathbf{R}^{2}, \underline{C}$. R. Acad. Sci. Paris, Ser. I 9 (1985), 395-398.

6. WEINSTEIN, A. Periodic nonlinear waves on a half-line, Comm. in Math. Phys. $\underline{99}$ (1985), 385-388.

7. WEINSTEIN, A. Erratum, Periodic nonlinear waves on a half-line, Comm. in Math. Phys. $\underline{107}$ (1986), p. 177.

8. SMILEY, M. W. Breathers and forced oscillations of nonlinear wave equations on $\mathbf{R}^{3}$, Journal für die reine und angewandte Mathematik 398 (1989), 25-35.

9. SMILEY, M. W. Time-periodic solutions of wave equations on $\mathbf{R}^{1}$ and $\mathbf{R}^{3}$, Math. Methods in Appl. Sci. 10 (1988), 457-475.

10. KATO, T. Perturbation Theory for Linear Operators, 2nd ed., Springer-Verlag, New York, 1976.

11. ADAMS, R. A. Sobolev Spaces, Academic Press, New York, 1975.

12. LIONS, J. L. and MAGENES, E. Nonhomogeneous Boundary Value Problems and Applications, Vol. I, Springer-Verlag, New York, 1972.

13. HARTMAN, P. Ordinary Differential Equations, John Wiley \& Sons, Inc., 1964.

14. PALEY, R. and WIENER, N. Fourier Transforms in the Complex Domain, A.M.S. Colloquium Publications, Vol. 19, Providence, R.I., 1934.

15. RUDIN, W. Real and Complex Analysis, 2nd edition, McGraw-Hill, New York, 1974.

16. ABRAMOWITZ, M. and STEGUM, I.(eds.), Handbook of Mathematical Functions, Dover Pub., New York, 1965.

17. WATSON, G. A Treatise on the Theory of Bessel Functions, Cambridge University Press, 1922.

18. RUDIN, W. Functional Analysis, McGraw-Hill, New York, 1973. 


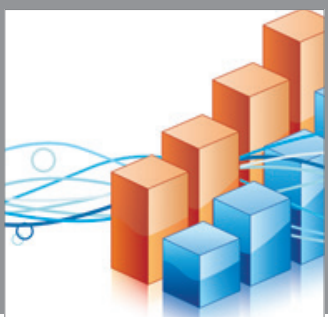

Advances in

Operations Research

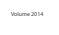

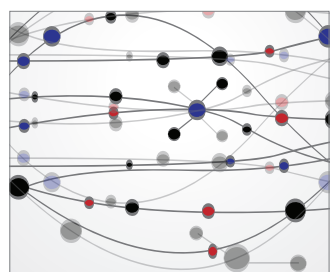

\section{The Scientific} World Journal
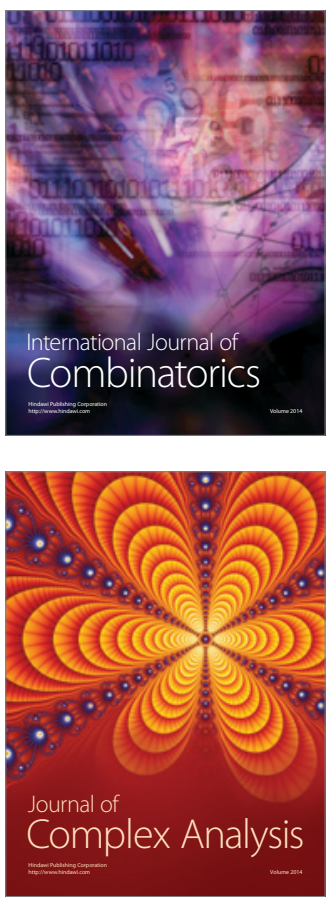

International Journal of

Mathematics and

Mathematical

Sciences
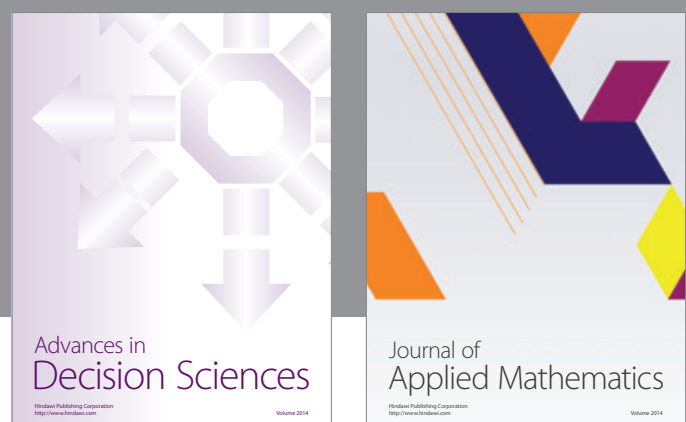

Journal of

Applied Mathematics
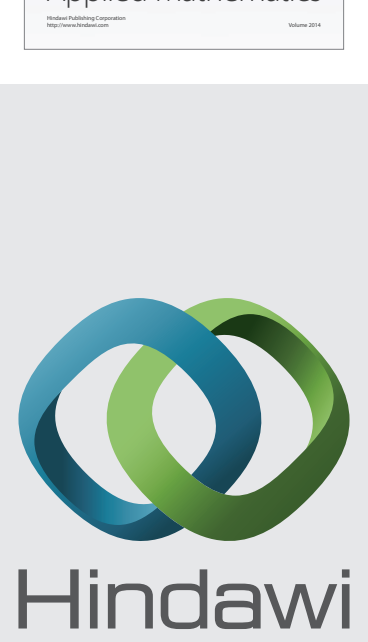

Submit your manuscripts at http://www.hindawi.com
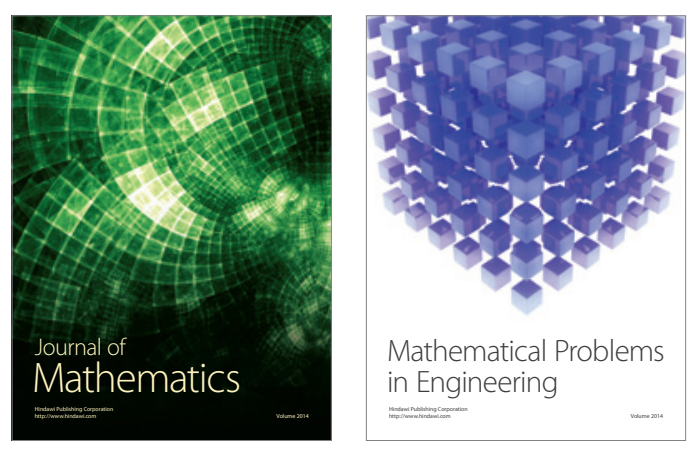

Mathematical Problems in Engineering
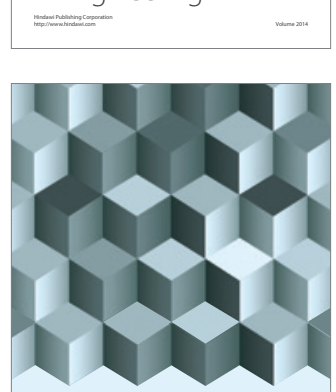

Journal of

Function Spaces
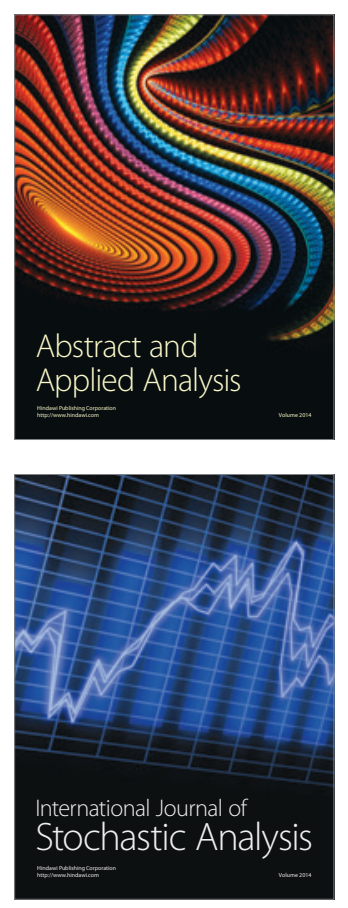

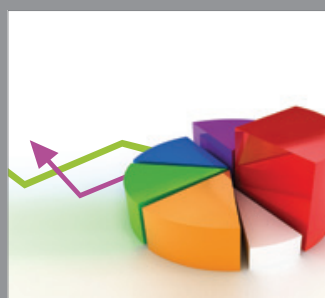

ournal of

Probability and Statistics

Promensencen
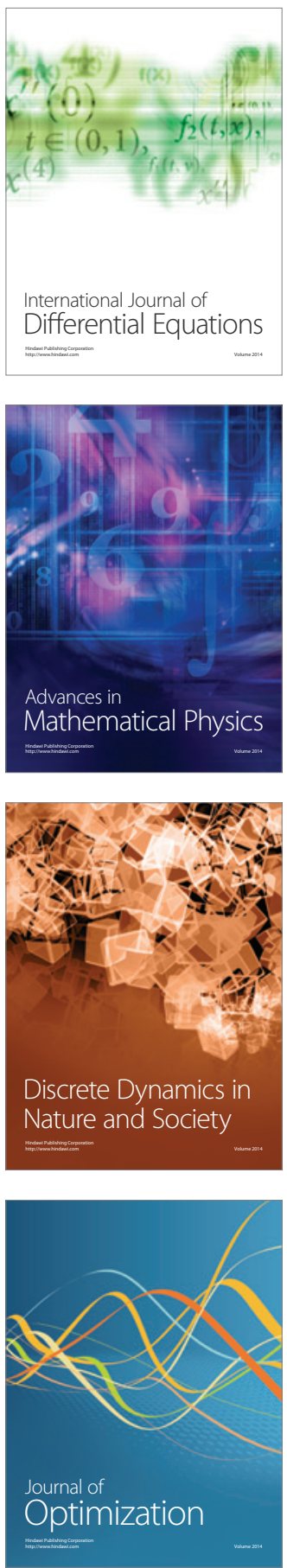